Selective Src-Family B Kinase Inhibition Promotes Pulmonary Artery Endothelial Cell Dysfunction.

\title{
Author Block:
}

5

Adam M. Andruska ${ }^{1,2}$, MD MSE

Md Khadem Ali ${ }^{1,2}, \mathrm{PhD}$

Xuefei Tian, MD, MS

Edda Spiekerkoetter ${ }^{1,2}, \mathrm{MD}$

\section{Author Affiliation:}

1 Division of Pulmonary, Allergy, and Critical Care, Stanford Medical School, Stanford, California 2 Vera Moulton Wall Center for Pulmonary Vascular Disease, Stanford University, Stanford, California.

\section{Keywords:}

1. Src-Family Protein Tyrosine Kinases

2. Endothelial Cell Dysfunction

3. BMPR2

4. Lck

5. Fyn

6. Tyrosine Kinase Inhibitors

7. NF- $\kappa \beta$ Signaling

\section{Abbreviations:}

Activin receptor-Like Kinase 1

2. BMPR2

Bone Morphogenetic Protein Receptor Type 2

3. BRE

BMP Response Element

4. DEG

Differentially Expressed Gene

5. EC

Endothelial Cell

6. ECD

Endothelial Cell Dysfunction

7. FYN

FYN Proto-Oncogene 
8. HTS

9. ID1,2, and 3

10. LCK

11. PAEC

12. $\mathrm{PAH}$

13. PBMC

14. PDGFR

15. pSMAD1

16. PBS

17. PTK

18. ROS

19. scRNA-seq

20. siRNA

21. si-Fyn

22. si-Lck

23. SRC

24. TCR

25. TKI
High Throughput siRNA Screen

Inhibitor of Differentiation 1, 2, and 3

Lymphocyte specific protein tyrosine kinase

Pulmonary Artery Endothelial Cell

Pulmonary Arterial Hypertension

Peripheral Blood Mononuclear Cell

Platelet derived growth factor receptor

Phosphorylated SMAD-1

Phosphate Buffered Saline

Protein Tyrosine Kinase

Reactive Oxygen Species

Single Cell RNA Seq

Small Interfering RNA

PAECs subject to Fyn knockout with si-RNA

PAECs subject to Lck knockout with si-RNA

Cellular Sarcoma Related*

T-Cell Receptor

Tyrosine Kinase Inhibitor

Vascular Endothelial Growth Factor Receptor 


\section{Abstract:}

55 Protein tyrosine kinases (PTKs) are essential for eukaryotic signaling. By targeting select PTKs, the group of drugs known as Tyrosine kinase inhibitors (TKIs) have proven to be effective for treating multiple diseases ranging from cancer to pulmonary fibrosis. However, some TKIs also paradoxically lead to the development of adverse conditions such as pulmonary arterial hypertension (PAH) by promoting endothelial cell dysfunction (ECD). We hypothesize that (1) subsets of PTKs may disproportionately modulate signaling pathways critical for endothelial homeostasis, such as BMPR2 signaling, and (2) inhibiting those pro-endothelial PTKs can promote the development of ECD. Herein we use an agnostic high-throughput siRNA screen to investigate how PTKs affect the canonical BMPR2 signaling pathway. Our major finding is that within the Src-family of nonreceptor PTKs, the Src-B family promotes canonical BMPR2 signaling while the Src-A family suppresses it. We focus on two representative members of each family, Lck (for Src-B) and Fyn (for Src-A) that are the strongest activators or inhibitors of BMPR2 signaling in the screen. We confirm that Lck is expressed in the endothelium of pulmonary arteries and show that Lck knockout (termed si-Lck) in pulmonary artery endothelial cells (PAECs) suppresses canonical BMPR2 signaling while Fyn knockout (termed si-Fyn) promotes canonical BMPR2 signaling. Furthermore, Lck and Fyn are responsible for opposing functional behaviors in PAECs: siLck promotes apoptosis and interferes with tube formation while si-Fyn suppresses apoptosis and promotes tube formation. After analyzing the whole-transcriptome signature of si-Lck and si-Fyn PAECs we find that in addition to BMPR2 signaling suppression, si-Lck (and not si-Fyn) increases a broad number of ECD markers and increases canonical NF- $\kappa \beta$ signaling. In summary, for the first time we show that Src-A and B Family of PTKs exert differential control over key endothelial cell signaling pathways resulting in direct phenotypic consequences. This knowledge may help to guide the design of more precise TKIs which avoid adverse drug reactions brought about through endothelial cell dysfunction. 


\section{Introduction:}

A distinguishing feature of eukaryotic cells is the evolution of protein kinases that coordinate inter- and intracellular signal transduction. Of the 518 protein kinases present in humans, the largest group (90) are Protein Tyrosine Kinases (PTKs) which further subdivide into receptor (e.g. VEGFR) and non-receptor, "cytoplasmic" (e.g. SRC, FYN, LCK) PTK families [1]. Whereas receptor-PTKs enable intercellular ligand-based communication, cytoplasmic-PTKs amplify signal transduction pathways based on external and internal cues controlling key functions such as proliferation and motility. Several non-receptor cytoplasmic PTKs are oncogenes (e.g. BCR-ABL), a fact that has driven the initial development of numerous Tyrosine Kinase Inhibitors (TKIs) which are now approved for conditions ranging from leukemia to pulmonary fibrosis [2]. However, though successful in controlling malignancy, TKIs have mixed effects in pulmonary vascular biology.

Pulmonary arterial hypertension $(\mathrm{PAH})$ is a progressive obliterative vasculopathy of the small pulmonary arterioles characterized by endothelial cell dysfunction (ECD) and apoptosis in concert with smooth muscle cell proliferation, vasoconstriction, and thrombosis [3-6]. The TKI Imatinib, mainly acting on the PTKs PDGFR and c-Kit, suppresses smooth muscle cell proliferation and improves clinical PAH as measured by 6-minute walk test distance [7, 8]. Paradoxically, newer generation of TKIs such as Dasatinib [9-11], Bosutinib [12], and Panotinib [13] are either known causes of PAH or are implicated in PAH development. Studies specific to

95 Dasatinib show this may be because of its action on a broader cohort of tyrosine kinases outside of PDGFR and c-Kit $[10,14,15]$, by direct induction of reactive oxygen species (ROS) in pulmonary artery endothelial cells (PAECs) [11], or by impairing endothelial cell (EC) barrier permeability in a Rho-kinase dependent manner $[16,17]$. In support of the notion that Dasatinib acts on a broader, potentially pathologic, set of PTKs is a recent pharmacovigilance study showing that specific cytoplasmic PTKs such as LCK, LYN, and YES are disproportionately associated with the development of $\mathrm{PAH}[18]$.

Despite the association of TKIs and their target PTKs with PAH, knowledge of how cytoplasmic PTKs act on specific cell types and specific signaling pathways continues to evolve. Since only 518 kinases phosphorylate 119,809 kinase targets [19], PTKs must be regulated in a way to prevent the unintended activation of cell 
expression. However, contemporary studies show that in environments where PTKs are in close spatial proximity (such as with LCK and FYN in the T-Cell Receptor complex) they preferentially act on different protein substrates, a property derived in part by the allosteric charge of their kinase pocket [20]. Because the affinity of a given TKI to the kinase pocket is also controlled by allosteric charge, it is reasonable to suspect that a given TKI may preferentially interact with a subset of PTKs and in turn block the phosphorylation of a specific subset of kinase targets. Since it is observed that (1) there is a disparate effect by TKIs on clinical phenotypes and (2) specific PTKs are singled out in human disease [18], we theorized that individual PTKs may hold an inherent bias on core signaling pathways critical for maintaining pulmonary vascular integrity. When blocked, these PTKs may enable or inhibit pathways critical for PAH pathogenesis.

One such core signaling pathway is the Bone Morphogenetic Protein Receptor 2 (BMPR2) pathway. Mutations in BMPR2 are the most common cause of hereditary PAH [4, 21]. In vitro silencing of BMPR2 recapitulates many features of ECD [22]. However, heterozygous mutations in BMPR2 have a disease penetrance of only $20 \%$, implying other genetic and environmental factors synergize with impaired BMPR2 signaling to realize disease [4]. Prior work investigating the interaction of PTKs with BMPR2 signaling shows that inhibition of the PTK SRC can improve BMPR2 signaling via improving BMPR2 receptor localization to the plasma membrane $[23,24]$. Additionally, SRC inhibition alone has no significant effect on Pulmonary Artery Endothelial Cell (PAEC) apoptosis [11]. We recently identified that lymphocyte specific protein tyrosine kinase (LCK), a gene coding for the Src-family tyrosine kinase LCK is a "BMPR2 activating" gene with the potential to support BMPR2 signaling [25]. Since our work with LCK is somewhat contradictory to that done with SRC - namely that LCK inhibition impairs BMPR2 signaling and SRC promotes it - we hypothesized that non-receptor tyrosine kinases might have an inherent differential effect on BMPR2 signaling. To test this hypothesis, we re-examined a broad group of PTKs in a high-throughput screen and found extensive heterogeneity within the Src-family of nonreceptor protein tyrosine kinases with respect to canonical BMPR2 signaling. Herein we show that PTKs divide into BMPR2-supportive and repressive- groups that respect the same evolutionarily defined boundary that distinguishes Src-Family-A from Family-B PTKs. Within these groups, we found that LCK of Src-Family B was the most BMPR2 activating kinase while FYN of Src-Family A was the most BMPR2 repressive. Further, we find that this division is not only applicable to BMPR2 signaling, but also results in differential control of the 
bioRxiv preprint doi: https://doi.org/10.1101/2021.09.27.462034; this version posted September 28, 2021. The copyright holder for this preprint (which was not certified by peer review) is the author/funder. All rights reserved. No reuse allowed without permission.

canonical NF- $\kappa \beta$ pathway. This work demonstrates that in endothelial cells of the lung, Src family kinases split L35 into two groups, a pro-BMPR2 anti-inflammatory Src family B group, and an anti-BMPR2 pro-inflammatory Src family A group. This knowledge may aid to better inform the design and selection of tyrosine kinase inhibitors which achieve therapeutic goals while avoiding the propagation of ECD. 


\section{Results:}

\section{A High-Throughput Screen Demonstrates that Src-Family A PTKs are BMPR2-Repressive, and Src-}

\section{Family B PTKs are BMPR2-Activating.}

As previously described, we employed a $\mathrm{C} 2 \mathrm{C} 12$ mouse myoblastoma cell line stably transfected with a BMP response element Luciferase Id1 (BRE-Luc-Id1) reporter [26]. Briefly, the BRE-Luc-Id1-reporter cell line was systematically transfected with a murine-wide siRNA library targeting 22,124 genes and then treated with L45 BMP4. Id1-linked luciferase expression was quantified by luminescence (Figure 1A). Simultaneously, cell viability was assessed with a tryptan-blue viability stain. To calculate the change in luminescence and viability for each well, luminescence and viability stain intensity was normalized to the overall average luminescence and viability stain intensity of the plate. This assumes that there are few "hits" (e.g. significant changes in Id1 or cell viability) per 384 well plate and is a standard method to normalized for plate-to-plate batch effects in a large, multi-plate high throughput assay. The average change in Id1 expression and cell viability relative to the plate baseline in response to knockout of one of the 22,124 genes across three replicates was calculated, which is shown in aggregate in Figure 2B. Genes to the left of 0 on the x-axis reduce Id1 expression when targeted by si-RNA while genes to the right of 0 on the x-axis increase Id1 expression after knockout. Using published phylogenetic classifications of receptor- and non-receptor-PTKs [1], representative PKTs from each major family were chosen and highlighted within the HTS results (Figure 1B, red dots) demonstrating that there are subdivisions of PTKs that activated or repressed Id1 expression. Plotting the change in Id1 expression relative to major representative PTKs (Figure $1 \mathrm{C}$ ) repeated this heterogeneity, but importantly showed the largest magnitude of change was seen with LCK and FYN, both members of the Src-Family of protein tyrosine kinases. Focusing specifically on the Src-Family of PTKs (Figure 1D), revealed that the magnitude of change in Id1 expression (an increase versus decrease) induced by PTK knockout in our HTS data matched exactly the established division between Src-A and Src-B kinases (Figure 1D and 1E). In summary, our agnostic, high throughput screen shows that knockout of Src-A family increases Id1 expression whereas knockout of Src-B kinases decreases Id1 expression. 
We next sought to determine if the Id1-PTK relationship seen in Figure 1D extended beyond the immortalized C2C12 cell line and into primary human cell lines. The first step was to identify an appropriate cell type in which the relationship between Src-A PTKs, Src-B PTKs, and BMPR2 could be studied. First, we decided to focus on

Lck as the representative Src-B family kinase, and Fyn as the representative Src-A family kinase. Dogmatically, Src-A kinases are widely expressed while Scr-B kinases are restricted to specific lineages [1]. Specifically, Fyn has been studied in endothelial and smooth muscle cells $[27,28]$ while Lck is traditionally believed to exist in T-Cells and to a lesser degree NK-Cells. It is only more recently that we and other have shown Lck is expressed in PAECs and HUVECs, respectively [25, 29]. It remained unknown if Lck was present at a protein l75 or message level in the pulmonary endothelium of human lungs, or if it was altered in disease. First, single molecule RNA in situ hybridization (sm-FISH) combined with immunofluorescent staining of human lung revealed that Lck mRNA was expressed at low levels in the endothelium, relative to high expression in Acta2/Cdh5 negative cells presumed to be lymphocytes, Figure 2A. Immunofluorescent staining of control human lung tissue demonstrated mild levels Lck within the endothelial layer with, as expected, strong expression in surrounding lymphocytes (Supplemental Figure 2). Lck protein was identified in cultured human PAECs (Figure 2B) with perinuclear localization consistent with its function as a cytoplasmic kinase. Human pulmonary artery endothelial cells obtained from both PAH patients and donor controls (Patient Characteristics are provided in Supplemental Table 1) also expressed Lck at a protein level, but in a heterogeneous fashion (Figure 2C). In addition to Lck, we also assessed for Id1 and Snail/Slug expression as surrogates for active L85 BMP and TGF- $\beta$ signaling. We found that there was a significant correlation between Id1 and Lck irrespective of whether the sample was obtained from control or PAH patients $(r=0.68, p=0.043$, Figure 2D). Though not significant owing to our low sample size, it is interesting to note that Id1 and Snail/Slug appear to have positive correlation in healthy control PAECs and a negative correlation in PAH PAECs (Supplemental Figure 2). Finally, publicly available single cell RNA sequencing data of Peripheral Blood Mononuclear Cells (PBMCs) from healthy controls was used to determine the expression of Lck along with members of the BMPR2 signaling pathway (Both Supplemental Figure 2 and main Figure 2E). This recapitulated the elevated expression of Lck in T-Cells and NK-Cells. However, BMPR2 and Id1 was expressed in few cells $(<20 \%)$ and at low levels, with the exception of dendritic cells. Though this data cannot comment on protein expression, it does imply that there is poor co-expression of $L c k, I d 1$, and BMPR2 in lymphocytes, and that the inherent low levels of $B M P R 2$ 
and Id1 expression at a basal state impairs our ability to detect meaningful decreases in expression of these genes. Because several studies have established that FYN is expressed in human PAECs [28] we concluded that the pulmonary artery endothelium would be the best initial location to study a potential interaction between BMPR2, Id1, Lck, and Fyn in the human lung. Finally, an effort was undertaken to investigate the expression of Lck in both mouse and rat endothelial cells. Despite using several antibodies and tissue preparation techniques, we were unable to find reliable expression of Lck in the endothelium of mice or rats (data not shown), as opposed to human endothelial cells.

\section{Suppression of LCK (a Src-B kinase), but not FYN (a Src-A kinase) significantly impairs basal and BMP9-mediated canonical BMPR2 signaling.}

!05 It is established that Lck and Fyn are essential components of the T-Cell Receptor (TCR) signaling pathway. Despite sharing significant similarity in amino acid sequence, and despite existing in close spatial proximity, LCK and FYN avoid phosphorylating each other's phospho-targets within the TCR, an important property theorized to avoid over- or under-activation of the TCR [30]. Recently, it was shown that the kinase pockets of Src-A and Src-B kinases are structured such that Src-B kinases (like Lck) are more tolerant of a positively charged amino acid environment surrounding the target tyrosine, whereas Src-Family A (like Fyn) prefer a more negatively charged environment [20]. This, combined with prior data showing immunoprecipitation between the SH3 region of Lck and SMAD proteins [31], led us to hypothesize that the discordant response of LCK and FYN on Id1 expression may be mediated by their actions on BMPR2 second messenger SMAD proteins. We first silenced LCK in PAECs using siRNA (hereafter termed "si-Lck" PAECs) and verified the knockdown by RT-qPCR (Figure 3A) and western blot (Figure 3B). Id1 expression by RT-qPCR significantly decreased in PAECs in non-serum reduced media (Figure $3 A, p=0.038$ ) while there was a significant upregulation in Snai1 expression (Figure 3A, $p<0.0001$ ). When starved PAECs were stimulated with BMP9 for 1.5 hours, there was an expected increase in phosphorylated SMAD-1 (pSMAD1) and Id1 (Figure 3C, 2Way Anova $p<0.0001$ for each). Si-Lck PAECs exhibited significant reduction in BMP9 induced Id1 expression (Figure 3C, 2-Way Anova $p=0.0013$ ) with the reduced level not significantly different from basal levels. Similarly, pSMAD1 was reduced $(p=0.0145)$ but not fully to baseline $(p=0.0208$ vs. basal state). Though there was a trend towards an increase in Snail/Slug expression with si-Lck, the effect was variable and not statistically significant at a protein level. There was no significant change in BMPR2 or total SMAD1 
expression across all conditions. Finally, pSMAD3 levels trended upwards with either si-Lck or with TGF- $\beta 1$

stimulation but were mild and not significant. This may be attributable to the relatively short treatment duration (Both TGF- $\beta 1$ and BMP9 were given 1.5 hours prior to cell harvest).

Turning attention to the Src-Family A kinase Fyn, we found that silencing FYN (hereafter termed "si-Fyn" PAECs) in non-reduced serum media had no effect on the expression of Id1 expression by RT-qPCR (data not shown). However, when starving cells in $0.2 \%$ FBS for 24 hours, si-Fyn induced a significant increase in Id1 expression (Figure 4A, Fold change 2.5, p<0.001) and BMPR2 (Fold change 1.6, p<0.001) 48 hours post transfection. We were unable to observe a significant change in pSMAD1 or Id1 induced by si-FYN in PAECs grown in starvation (0.2\% FBS) conditions at 72 hours. The effect of si-FYN was variable in PAECs grown in full media (2\% FBS) conditions. However, there was a significant increase in BMP9 mediated Id1 expression

!35 (Fold Change 1.6, 2-Way Anova $p<0.01$, Figure 4-B,C). Also, BMP-9 treated si-FYN PAECs had a significant decrease in pSMAD3 expression (Fold Change 0.4, 2-Way Anova $p<0.001$ ) relative to nontargeted controls. We were unable to detect a change in SNAIL/Slug expression at 72 hours in si-Fyn cells no matter the media conditions. Finally, the effect of TGF- $\beta 1$ with si-Fyn was investigated (supplemental Figure 4). Knockout of FYN in full media conditions increases phospho-SMAD 1 activity, even in the presence of TGF- $\beta 1$.

Taken together, this data shows that Lck and Fyn have opposing effects in the pulmonary endothelium with respect to canonical BMPR2 signaling. Absence of Lck decreases SMAD1 phosphorylation and Id1 expression, while FYN knockout promotes BMP9 mediated SMAD1 phosphorylation and Id1 expression.

\section{LCK (a Src-B kinase) but not FYN (a Src-A kinase) Suppression in human pulmonary artery} endothelial cells is associated with endothelial cell dysfunction.

We next sought to determine if suppression of Lck or Fyn generated a phenotypic difference in endothelial cells. Tube formation is an established BMPR2-dependent assessment of endothelial phenotype. Si-Lck and si-Fyn PAECs were examined alongside non-targeted siRNA treated PAECs. Note that the optimal concentrations of siRNA and lipofectamine required to achieve an effective knockdown of Lck in PAECs is different than the optimal concentrations required to achieve Fyn knockdown. Therefore, si-Lck and si-Fyn conditions are compared to controls termed si-NT-F and si-NT-L that use matching siRNA and lipofectamine 
concentrations. 48 hours after knockdown, viable cells were passaged, counted, and seeded on Matrigel. SiFyn produced a significantly higher total tube length with more tube junctions relative to si-NT-F (Figure 5A-B).

!55 Conversely, si-Lck resulted in PAECs that sprouted few short tubes compared to si-NT-L. Total tube length and number of tube junctions was significantly less in si-Lck versus the si-NT-L (Figure 5 A-B). Notably, cell death was accelerated after si-Lck versus si-NT-L (Figure 5-C). We hypothesized that the observed cell attrition correlated to an increase in apoptosis. Assessing Bax and $B c$ gene expression by qPCR revealed higher Bax and $\mathrm{Bcl}$ levels si-Lck cells versus si-Fyn cells relative to their respective controls (Figure 5-D). Increased apoptosis was again seen when assessing Caspase 3/7 activity (Figure 5-E). Finally, definitions of endothelial cell dysfunction include the upregulation of expression of integrins along with other pro-inflammatory cues. To assess this, 72 hours after Lck or Fyn knockout in full (2\% FBS) media conditions, VCAM1 protein expression was assessed (Figure 5-F). Here we found increased VCAM1 expression si-Lck cells but low levels in si-Fyn cells. Taken together, this data suggest that suppression of the Src-B kinase Lck disrupts normal endothelial cell function, but suppression of Fyn does not. Si-Lck PAECs fail to form tubes, exhibit higher rates of apoptosis, and increase expression of the cells surface integrin VCAM1, a maker associated with endothelial cell dysfunction.

\section{Whole transcriptome analysis of PAECs subject to Lck and Fyn knockdown validates a differential}

effect on BMPR2 signaling and reveals that Lck knockout confers a pro-inflammatory signature as compared to Fyn knockout centered on NF-k $\beta$ canonical signaling.

To this point, we have taken a narrow view of the action of FYN and Lck in PAECs that is centered on canonical BMPR2 signaling. We know that each PTK engages thousands of tyrosine phospho-targets in eukaryotic cells. Therefore, it is unlikely that Lck and Fyn in PAECs are only acting on BMPR2 signaling. In order to determine !75 if additional signaling pathways outside of the canonical BMPR2 signaling pathway are differentially regulated by Lck and Fyn and therefore potentially involved in mediating endothelial dysfunction, we performed a transcriptome-wide assessment of PAECs subjected to si-Fyn or si-Lck by RNA seq. After performing the initial differential gene expression analysis, we curated a specific set of genes genes that were differentially regulated by both Lck and Fyn in opposing ways. 
We performed bulk-cell RNA sequencing of four groups of PAECs: si-Lck, si-Fyn, a scrambled siRNA transfection group (NT), and a scrambled siRNA group subjected to BMP9 stimulation (NT+BMP9). Note that in this case, we used similar concentrations of siRNA and lipofectamine in all groups to control for gene expression variability induced by the transfection process. Although this resulted in a less stringent Lck knockdown condition relative to Fyn, we felt that RNA sequencing would be able to adequately detect patterns in gene expression change in this less stringent knockdown condition. We additionally transfected a group of PAECs with scrambled siRNA and treated with $50 \mathrm{ng} / \mathrm{mL}$ of BMP9 for 2 hours to activate the canonical BMPR2 signaling pathway (BMP9 group). Three replicates per condition were used. Knockout of target genes was verified by RT-qPCR prior to performing RNA sequencing and did indeed verify Fyn and Lck knockdown (Fyn $F C=0.13, p<0.001, L c k F C=0.15, p=0.004)$. Quality control metrics for the RNA-seq analysis are shown in Supplemental Figure 5-A. Principal component analysis (PCA, Supplemental Figure 5-B) showed that each within-group samples clustered closely together. Further, PCA analysis showed appropriate separation between each group. Volcano plots of DEGs in the si-Lck and si-Fyn knockout conditions are shown in Figure 6A, reproduced in larger format in Supplemental Figures 6 and 7. It should be noted that although Fyn was identified in the DESEQ2 analysis as a significantly downregulated gene in the siFyn case, Lck was not found to be significantly downregulated in the si-Lck case. This is because the raw counts for Lck are relatively low in PAECs resulting in the dropout of Lck during DEG analysis. The volcano plot for the siNT+BMP9 condition is shown in supplemental Figure 8. From the si-Lck volcano plots, notable upregulated genes included $/ / 6 s t$, Ccl7, Wnt5a, and I/1 3 . Notable downregulated genes were Ace, Lyve1, Gja5, and Dysf. In the si-Fyn cases TGF- $\beta$ and endothelial cell-specific genes which were upregulated included Bmp4, Gdf7, Gdf11 (Bmp11), Gja5, Lyve1, Id1, and Tgfb1. Genes downregulated in the si-Fyn case include TIr3, C1s, C3, Ccl8, and Vcam1.

From the heatmap in Figure 6B, we identified clusters of genes both upregulated in the si-Fyn condition and the siNT+BMP9 condition but downregulated in the si-Lck condition. This block includes the known BMP9 responsive genes Id1, SMAD6, Hey2, and BMPR2 [32]. Outside of this block was a large group of genes upregulated in the si-Lck condition and downregulated in the si-Fyn and siNT+BMP9 condition. These genes correlated with both inflammation and endothelial cell dysfunction, and included Vcam1, Icam1, IL-1 $\beta$, IL-6, and Ccl8, among others [33]. We went on to specifically analyze the DEG data for genes known to be critical in 
endothelial cell biology. In Figure 6C, fold change and p-values of selected genes know to confer endothelial identity, ECD, endothelial health, apoptosis, BMPR2 signaling, and NF- $\kappa \beta$ signaling are shown. Notable is the downregulation of endothelial specific marker genes (Pecam1, Gja5, Cdh5, Cldn5) [34] in si-Lck PAECs and upregulation of the same genes in si-Fyn PAECs. Genes implicated in endothelial to mesenchymal transition (EndMT) were upregulated in si-Lck (MMP9, COL1A1) and downregulated in si-Fyn (Twist1) PAECs [35]. This dichotomy continued for genes associated with ECD (Cc/8, Cx3c/1, IL-1B, HLA-A, Vcam1, Icam1), genes associated with endothelial health such as Prostaglandin Synthases (Ptgs1, Ptgis) or Nitric Oxide Synthase (Nos3), and genes associated with apoptosis (Bak, Fas). Taken together, this set of data suggests that si-Lck and si-Fyn conditions induce significant and opposing changes in gene expression correlating with endothelial cell identity and function.

320 Given this dichotomy, we investigated the set of genes that were both alter by si-Lck and si-Fyn conditions but in an inverse manner - in other words, genes that were significantly upregulated in the si-Lck condition and significantly downregulated in the si-Fyn condition or vice versa. Note that a lower fold change of greater than 1.25 or less than 0.8 with a false discovery rate of less than $5 \%$ was used because (1) some genes may not produce as large a fold change in the si-Lck condition as compared to the si-Fyn condition and (2) we wanted 325 to capture a larger set of genes for downstream analysis. In Figure 6D the Venn diagram shows that there are 671 total genes which are both significantly upregulated by si-Lck and are significantly downregulated by siFyn (Figure 6E). There are 481 genes which are both significantly downregulated by si-Lck and significantly upregulated by si-Fyn (Figure 6F). To determine how these sets of DEGs correlated with cellular functions, gene set enrichment analysis was performed [36]. In parallel, the TRRUST, BART, and oPOSSUM-3 packages were used to identify transcription factors predicted to act on the set of differentially expressed genes [37-39].

First, in the "siLck Up" / "siFyn down" group (Figure 6E, reproduced in larger format in Supplemental Figure 9), we found that the genes most differentially regulated included Tnfaip6 and I/1B, genes associated with the type-1 interferon signaling pathway. Gene ontology analysis in the siLck Up/siFyn Down group indicates activation of pathways typically elicited by interferon gamma or lipopolysaccharide stimulation, correlating to increased interferon $\alpha / \beta$ production, IL-6 production, NF- $\kappa \beta$ activation, and MHC-1 activation. Indeed, when 
performing transcription factor prediction by the three methods noted above, the canonical NF- $\kappa \beta$ transcription factor, RelA, was strongly implicated as being activated by si-Lck (Figure 6E). Returning to tissue culture, it was indeed found that phosphorylated p65-RelA was significantly increased in the nuclei of PAECs subject to Lck knockout compared to Fyn knockout or nontargeted (NT) controls (Figure 6G). In total, the siLck up/siFyn down group of genes is felt to represent a "Pro-Inflammatory" gene signature which is dominated by canonical NF- $\kappa \beta$ activity.

Next, in the "siLck Down" / "siFyn Up" group (Figure 6F, Supplemental Figure 10), opposingly regulated and 345 differentially expressed genes consisted of endothelial artery and capillary specific genes (Gja5, Lyve1, Gphibp1). Gene ontology analysis correlated with pathways specific to sprouting angiogenesis, endothelial cell migration, and adherent junction organization. Transcription factor analysis did not predict one single transcription factor, but did implicate Snai1, Sp1, and Erg. Snai1, a critical marker of EndMT, was already shown to be increased by Lck knockout in Figure 3A. Erg has recently been shown to both be decreased in pulmonary hypertension [40] and exert control over Notch signaling to promote vascular stability [41]. Sp1 activates VEGF expression in tumor derived endothelial cells. Also, Sp1 acts as a promoter for the gene Endoglin. Loss of Sp1 can contribute to the development of HHT and PAH due to Endoglin suppression [42]. Taken together, the set of differentially expressed genes shared by Lck and Fyn (Lck Down/Fyn Up) share features of endothelial identity and includes BMPR2 based canonical signaling activity. Thus, this set of genes is termed a "Endothelial Identity" gene set.

Finally, we attempted to find how the gene signatures of the siFyn and siLck overlapped with the gene signature generated by BMP9 treatment in PAECs. We made the initial assumption that genes upregulated by BMP9 treatment represent a desired gene signature for endothelial health [43]. Because FYN knockdown confers a pro-endothelial, anti-inflammatory phenotype and transcriptomic signature, we focused on genes that were coregulated in the same direction by BMP9 and siFYN (e.g. genes downregulated with both siFYN and BMP9, Supplemental Figure 13, or genes upregulated with both siFYN and BMP9, Supplemental Figure 14). Because LCK knockout was pro-inflammatory and conferred anti-endothelial properties, we focused on genes that were regulated in opposite directions by BMP9 and siLCK (e.g. genes downregulated with siLCK and upregulated 
365 with BMP9, Supplemental Figure 11, or genes upregulated by siLCK and downregulated by BMP9 treatment, Supplemental Figure 12).

Genes upregulated by both siFYN and BMP9 included Id1, Lrrc4, II21r, Gja5, Smad6, Hey2, Cxxc5, Gli2, Sox8, Smad7, Foxs1, and Smad9. Aside many being implicated in the BMPR2 signaling pathway, Gene Ontology analysis strongly correlated with established BMP functions, including vascular smooth muscle cell differentiation (GO:0035886) and cardiac ventricle development (GO:0003231).

Genes downregulated by both siFYN and BMP9 included Cxc/8, Batf2, Mypn, Cxcl2, Stc1, Cebpd, Epn3, Cxcl1, Socs1, Tnfsf15, Cx3cl1, Apol6, Themis2, Tnfaip3, Sp6, and II1a. Selected results from Gene Ontology analysis 375 include B cell homeostasis (GO:0001782), Negative regulation of smooth muscle cell proliferation (GO:0048662), Response to lipopolysaccharide (GO:0032496), and Regulation of I- $\kappa \beta$ kinase/NF- $\kappa \beta$ signaling (GO:0043122).

Genes upregulated by BMP9 and downregulated by siLCK included Lrrc4, Smad6, Gja5, Nog, Nrarp, Sox18, Sema3G, Tmem37, Gprin3, Snai2, Dll4, Nfatc2, Bmf, Itgb4, Enc1, Pmepa1, Tspan13, Samd5, Myom3, Vahs1, Dhh, Cxcl12, Tmc7, Cpne5, Iffo2, Wnt9A, and Jag2. Selected results from Gene Ontology analysis include Mitral valve development (GO:0003174), Pharyngeal arch artery morphogenesis (GO:0061626), and negative regulation of pathway-restricted SMAD protein phosphorylation (GO:0060394).

385 Finally, genes downregulated by BMP9 and upregulated by siLCK include Pdk4, Cxcl2, Cxcl1, Cxcl8, Cebpd, Tnfaip3, Znf296, Txnip, Ntm, Ccl2, RGMB-AS1, Adm, Cd274, Cx3cl1, Lypd6, Socs1, Pcdh17, and Foxo3. Gene Ontology results include Regulation of transforming growth factor beta2 production (GO:0032909), PERK-mediated unfolded protein response (GO:0036499), regulation of cytokine production involved in inflammatory response (GO:1900015), and positive regulation of vascular endothelial growth factor production 390 (GO:0010575). Of the above genes, Lrrc4 and Smad6 appears to be co-regulated the most by BMP9, siLCK, and SiFYN. 
Taken together, whole transcriptome analysis of PAECs subject to Fyn and Lck knockout demonstrates that si-Lck and si-Fyn indeed co-regulate two different gene programs which reveal expanded functions beyond 395 that of regulating canonical BMPR2 signaling. The set co-regulated genes can be divided into two major programs. In the first program, termed the "Endothelial Identity" program, Lck suppression will reduce the expression of genes associated with endothelial identity whereas Fyn suppression will increase expression of the same set of genes. In the second program, termed the "Inflammatory" program, Lck suppression will increase the transcription of genes controlled by canonical NF- $\kappa \beta$ signaling, specifically those modulated by

the transcription factor RelA. This program also closely mimics the effect of treating PAECs with Interferon $\gamma$ or IL-1. Fyn knockout serves to suppress the "Inflammatory" program, directly counteracting the effect of RelA and canonical NF- $\kappa \beta$ signaling.

\section{Discussion:}

Historically, it was thought that Src-A and Src-B protein tyrosine kinases were distinguished based on cell-type expression, with Src-A kinases expressed in diverse cell types and Src-B being restricted to cells of hematopoetic origin [44]. Later phylogenetic analysis showed that Src-A and Src-B kinases were genetically distinct, diverging very early in eukaryotic evolution [1]. The advent of high throughput technologies has more recently shown that the Src-A and Src-B kinases can also be distinguished based on their kinase pocket amino acid sequence [20]. Further, the charges within the kinase pocket confer substrate specificity that results in each family of kinases preferring distinct and orthogonal peptide substrates, a feature that is suspected to prevent cross-talk between kinases and substrates in regions of close spatial proximity, such as within the TCell Receptor [45]. In this study we use pulmonary artery endothelial cells to show for the first time that H15 differential effects of Src-A and Src-B kinases extends to intracellular signaling pathways, in this case BMPR2 and $\mathrm{NF}-\kappa \beta$.

Starting with a high-throughput siRNA screen in an Id1 reporter cell line subjected to BMP4 stimulation, we show that Src-A PTKs are inherently Id1/BMPR2 suppressive, and Src-B PTKs are inherently Id1/BMPR2 activating. Two representatives of the Src-A and Src-B families, LCK and FYN, have the most significant effects on ID1 expression, with LCK knockout decreasing ID1 the most and FYN increasing ID1 the most. We show 
that both FYN and LCK are present in endothelial cells at both a protein level and an mRNA level. It is important to note that both FYN and LCK are present in high levels in lymphocytes. We chose to focus on the endothelium in this case for two reasons. First, our available data showed that transcripts from the BMPR2 signaling pathway 125 (e.g. Bmpr2 and Id1) were not expressed at high levels in lymphocytes, therefore making it hard to detect if manipulating PTKs reliably effects canonical BMPR2 signaling, as we have defined it. Second, the role of LCK in endothelial cells was not well established. It is possible that there are other BMP responsive type 1 and type 2 TGF- $\beta$ receptors present in lymphocytes that are regulated by Lck and Fyn which could be the subject of future studies. From the perspective of our public SC-RNA seq PBMC analysis, it seems that Id2 may be a better BMP-responsive transcription factor to study in lymphocytes as opposed to Id1. It remains an open question as to how LCK and FYN manipulate BMP/TGF- $\beta$ signaling in lymphocytes. Lck is known to be reduced in PBMCs of patients with interstitial lung disease [46], lupus [47], and, as we have shown, pulmonary arterial hypertension [25]. However, the results presented in this paper show that this more ubiquitous peripheral reduction of Lck in PAH patients does not translate universally to the endothelium of the human lung. We did 135 however observe a strong correlation between Lck and Id1 expression, implying that Lck may indeed facilitate BMP signaling in endothelial cells in a positive way.

Focusing on the two representative kinases, LCK and FYN, we sought to determine if this finding could be replicated in primary human cells. Silencing Lck in cultured pulmonary artery endothelial reduced Id1 mRNA with a concurrent upregulation of Snai1 mRNA, a transcription factor traditionally associated with TGF- $\beta$ activity. We show that Lck knockout nearly reduced BMP9 mediated Id1 protein expression to basal levels, with a significant, but less complete, reduction in phosphorylated SMAD1. The converse was seen with Fyn silencing. Here Id1 was increased at a transcriptional level at basal levels and BMP9 mediated Id1 expression was enhanced. Functionally, Lck and Fyn had dichotomous effects on cellular phenotypes, with Lck increasing apoptosis, impairing cell proliferation, blocking tube formation, and increasing markers of endothelial cell dysfunction such as VCAM1.

This study is significant for several reasons. First, it provides a link between Src-Kinases and TGF-Beta signaling that has not previously been demonstrated. Although previous studies have extensively studied Src- 
150 family PTKs in pulmonary hypertension, these studies focused primarily on smooth muscle cells [27, 48]. The current study investigates specifically pulmonary artery endothelial cells. Our results do provide a rationale from which to link a commonly used class of drugs (Tyrosine Kinases Inhibitors) to the development of endothelial cell dysfunction, specifically if the inhibitor disproportionately targets Src-B PTKs. Second, this study shows that Lck, a tyrosine kinase originally thought to be expressed only in Lymphocytes and more recently, in Schwann Cells [49], is also expressed in pulmonary endothelial cells. Finally, this study puts forth the concept that better understanding the dichotomous actions of Src-A and Src-B kinases may help better inform the design of more precise tyrosine kinase inhibitors. Admittedly, because each PTK has hundreds of potential targets which manipulate intracellular signaling in unpredictable ways, each individual PTK can be thought of as a node in a large intracellular signaling network. It is difficult to predict the phenotype created by blocking a single kinase node and altering a multitude of pathways. However, developing a better understanding of the protein substrates prefferred by Src-A and Src-B kinases in endothelial cells may improve how we approach manipulating the signaling network with tyrosine kinase inhibitors. Regardless, this study suggests that designing tyrosine kinase inhibitors with a low affinity for Src-B kinases may lessen the development of endothelial cell dysfunction as an adverse drug effect.

\section{Funding Support:}

This work is supported by NIH 1 R01 HL128734-01A1 Targeting Novel BMPR2 modifiers in Pulmonary Hypertension with Repurposed Drugs (E.S.) and NIH 5T32HL129970-02 Training Fellowship in Lung Biology (A.M.A.). 


\section{Methods:}

175 1. High Throughput siRNA Screen:

High throughput siRNA screening of $>22,000$ genes using an Id1-BRE luciferase reporter assay in a C2C12 mouse myoblastoma cell line treated with or without 250 pM BMP4 was conducted in the Stanford HighThroughput Bioscience Center, as previously described [50]. Briefly, the C2C12 myoblastoma cells, a generous gift from Dr. Peter ten Dijke, were stably transfected with BRE- Id1 linked to luciferase and used as a reporter cell line [26]. Cells were screened on 384-well siRNA plates. The transfection conditions were optimized with BMP4 as the stimulus, siBMPR2 as the positive control, and siTox (a toxic siRNA) as a final control. Cells were seeded in wells at a density of 1500 cells/well. Next, siRNA was transfected at an optimized concentration of $25 \mathrm{nM}$ using DharmaFect3. After 48 hours, cells were stimulated with 250 pM of BMP4. Two hours after stimulation, the change in luminescence of an individual well was measured relative to the average

185 luminescence of all wells in the plate. After this, a cell viability stain with tryptan-blue was performed. Transfections were performed in triplicate for each gene, with the end product being change in Id1 linked luminescence relative to baseline and change in cell viability relative to baseline.

\section{Cell Culture.}

190 Human PAEC (Promocell) were grown as monolayers in gelatin-coated dishes in a commercial EC (Promocell media. Cells were passaged at 1:3 ratios and used for experiments from passages 3-6.

\section{Human PAH Tissue: Isolation of Cells lung tissue.}

PAEC of IPAH and FPAH patients at time of lung transplant were obtained from digested whole lung tissue, 195 using CD31-AB pulldown beads (Dynabeads; Invitrogen), as previously described [50]. Experiments involving human tissue or derived primary cells were approved by the Stanford University Institutional Review Board and the Administrative Panel on Human Subject Research.

\section{RNA Interference.}


;00 Both Lck and Fyn expression was modulated by RNAi in PA endothelial cells (PAEC). A pool of 4 siRNAs for BMPR2, FHIT, LCK or a non-targeting control pool (Ambion, Table. 1) were transfected into PAECs using the RNAi Max kit (Invitrogen) for 48 hours. mRNA knockdown efficiency was determined by qPCR.

Table 1:

\begin{tabular}{ccc}
\hline Target Gene & Cat. \# & Sequence (Sense) \\
\hline Lck & Ambion 4392440 & GGAAUUAUAUUCAUCGUGAtt \\
Fyn & Ambion 4390824 & GAUUGAUAGAAGACAAUGAtt \\
Non-Targeted (NT) & Ambion 4390843 & Not published by Manufacturer \\
\hline
\end{tabular}

\section{5. qPCR Assay to Detect mRNA Expression.}

;05 For mRNA, total RNA was extracted from adherent cell layers using the RNAeasy Plus Kit (Qiagen) and reverse transcribed into cDNA using random primers with the Taqman cDNA reverse transcription Kit (Applied Biosciences) according to the manufacturer's instructions. Prior to the generation of cDNA, RNA quality and concentration was measured using the Nanodrop system (Thermo-Fisher). The level of mRNA expression was quantified using Taqman primer/probe sets (Table 2) for the target and normalized to a housekeeping control ;10 (18s). In order to validate new PCR probes, standard curves were calculated from serial 1:2 dilutions of cDNA across 6 different concentrations, and PCR probe efficiency $(E)$ was calculated. From the standard curve, the relative standard method was used to determine relative concentrations of mRNA in lysates.

\section{Table 2:}

\begin{tabular}{cc}
\hline Target Gene & Cat.\# \\
\hline Bmpr2 & Hs00176148_m1 \\
Id1 & Hs03676575_s1 \\
Fyi1 & Hs00195591_m1 \\
Gapdh & Hs00176628_m1 \\
Lck & Hs01786624_g1 \\
18s & Hs00178427_m1 \\
Bax & Hs99999901_s1 \\
Bcl & Hs00180269_m1 \\
\hline
\end{tabular}

\section{Western Blotting:}

;15 Western blotting was performed as previously described [50]. Antibodies were used as described in Table 3:

\section{Table 3:}




\begin{tabular}{cccccc}
\hline Target & Manufacturer & Cat. \# & Clone & Species & Dilution \\
\hline B-Actin & Santa Cruz & SC4778 & C4 & Mouse & $1: 600$ \\
BMPR2 & Invitrogen & MA5-15827 & 3F6F8 & Mouse & $1: 800$ \\
Lck & Abcam & Ab227975 & EPR20798-107 & Rabbit & $1: 300$ \\
pY-394 Lck & Abcam & Ab201567 & MM0795-71W27 & Mouse & $1: 500$ \\
Id1 & Santa Cruz & sc-133104 & B8 & Mouse & $1: 100$ \\
Snail/Slug & Abcam & Ab180714 & Polyclonal & Rabbit & $1: 500$ \\
VCAM1 & R\&D Systems & BB16-V1 & BBIG-V1 & Mouse & $1: 500$ \\
Fyn & BD Biosciences & BD61063 & 25/Fyn & Mouse & $1: 500$ \\
pSMAD-1/3 & Abcam & ab52903 & EP823Y & Rabbit & $1: 1000$ \\
SMAD1 & Cell Signaling & CST 9743 & Polyclonal & Rabbit & $1: 500$ \\
RelA & Cell Signaling & CST 8242 & D14E12 & Rabbit & $1: 1000$ \\
\hline
\end{tabular}

\section{Apoptosis and Matrigel Tube Formation Assays:}

Assays were conducted according to the manufacturer's instructions and as previously described [50, 51].

\section{RNA Sequencing:}

Human PAECs were grown to $80 \%$ confluency on gel coated plates and transfected with either Fyn, Lck, or nontargeted siRNA. At 48 hours, cells were placed in 0.2\% FBS starvation media. Two hours prior to harvest all cells were treated with either PBS in $0.2 \%$ FBS media or, in one case, $20 \mathrm{ng} / \mathrm{mL}$ of BMP9 in $0.2 \%$ FBS starvation media. Cells were harvested and mRNA was extracted via Qiagen mini columns. RNA quality was assessed with the Agilent 2100 Bioanalyzer system and found to have a RIN of 9.8-9.9 (Supplemental Figure 5-A). cDNA libraries were generated (Illumina), barcoded, and sequenced via a paired-end 150 bp sequencing strategy to a depth of between 38 and 46 million paired-end reads on the Illumina platform. Raw reads were mapped to the human genome using STAR [52]. Quality control metrics regarding sequencing and alignment are given in Supplemental Figure 5-A. Differential gene expression analysis was performed using the $R$ package DEseq2 [53]. Briefly, differentially expressed genes were calculated via the "DEseq" command after setting transfection condition (FYN, LCK, NT) and treatment (PBS, BMP9) as factors. Conditions were contrasted against the nontargeted PBS condition to determine the fold change and adjusted $p$-value for each gene in each condition. A variance stabilizing transformation of the raw count data was used to generate the gene expression heat map. Next, differentially expressed genes present in each condition were found using a 
$5 \%$ and the R function "venn.diagram". Lists of significant differentially expressed genes that were present in select conditions (IE increased in the LCK case and decreased in the FYN case) were used for gene ontology analysis. Gene ontology and transcription factor analysis was facilitated using the Enrichr GSEA web server $[36,54]$.

\section{Analysis of Public PBMC Single Cell RNA Seq Data:}

Raw count data of 2700 PBMCs (PBMCs from a health human donor, single cell immune profiling dataset by Cell Ranger 1.1 .0 on Illumina NextSeq 500 with $\sim 69,000$ reads per cell, 10x Genomics, 2016, May 26) were analyzed using the Seurat V 3.0 sc-RNA seq pipeline to perform unsupervised hierarchical clustering [55]. Average gene expression was calculated on a per-cluster basis. Cell identity was determined on the basis of differentially upregulated genes in a cluster of interest relative to all the other clusters in the dataset (Supplemental Figure 2). After clusters were developed and identified, the average expression of genes of interest (Bmpr2, Id1, Id2, Id3, Lck) was determined. The percent of cells expressing the gene of interest (defined as a cell with $>1$ count) was also calculated. Both the magnitude of expression (presented as a Z-score relative to all cells in the sample) and the percent expression are presented in dot plot format.

\section{Immunofluorescence Staining of Adherent Cells and Human Lung Tissues:}

For confocal localization of proteins in cells, cells were washed in warm PBS and then fixed in $10 \%$ normal buffered formalin for 20 minutes at room temperature. Cells were washed with PBS, then permeabilized and blocked with $0.3 \%$ Triton $\mathrm{X}-100$ and $5 \%$ Serum for $1-3$ hours at room temperature. Primary antibodies were incubated overnight at $4 \mathrm{C}$ at the following dilutions in a 5\% serum buffer in PBS: LCK (ABCAM Ab227975, 1:200), FYN (BD Biosciences BD61063 1:150), phopho-P65 RelA (Cell Signaling Technologies 93H1,1:200). Appropriate secondary antibodies were used at a 1:250 dilution in a 5\% serum PBS buffer for 1 hour. Cells were washed in PBS $+0.5 \%$ Tween-20 $\times 3$. In all cases DAPI counter stains or F-Actin counter stains ;60 (fluorescently labeled phalloidin) were used per published protocols. Cells were imaged by confocal microscopy. Quantification of signal was performed in ImageJ. 
For human tissues, sections were deparaffinized in Xylene per standard protocols and rehydrated. Antigen retrieval was performed in a $10 \mathrm{mM}$ Sodium Citrate buffer at $100 \mathrm{C}$ for 10 minutes. Tissue were permeabilized and blocked in a $0.3 \%$ Triton X-100 and 5\% goat serum buffer in PBS for three hours. Antibodies were incubated in a $5 \%$ serum, $0.3 \%$ Triton-X-100 buffer overnight at $4 \mathrm{C}$ at the following dilutions: LCK (ABCAM Ab227975, 1:100) and Acta2 (Direct Cy3 Conjugation, 1 A4 Clone, Sigma-Aldrich C6198, 1:200). An AlexaFluor 633 Goat anti-Rabbit Secondary antibody was incubated at 1:250 dilution in a 5\% serum, 0.3\% Triton X100 buffer in PBS for 1 hour at room temperature. Tissue was washed $x 3$ in PBS $+0.5 \%$ Tween-20. DAPI counter staining was performed. Due to the high amount of auto fluorescent structures in human lung, the 488 laser on the confocal microscope was used to collect light outside of the emission windows of Cy3 and AlexaFluor 633 in order to accurately capture the tissue's background autofluorescence. Cy3 and Alexa-Fluor 633 were imaged with narrow $(50 \mathrm{~nm})$ collection widows around their peak emission wavelength. Images were processed on ImageJ software.

\section{Single Molecule mRNA Fluorescent in situ Hybridization (sm-FISH):}

Sm-FISH for Lck was performed using the RNA-scope protocol [56] (Advanced Cell Diagnostics, Inc.) using the Channel 1 probe to Lck (Cat No. 440201). After completion of the sm-FISH protocol, tissue was counter stained for endothelial (VE-Cadherin, R\&D AF938, 1:300) and smooth muscle (Acta2, Direct a488 Conjugation, 1A4 Clone, 1:200). Tissue was imaged by confocal microscopy and analyzed with ImageJ.

\section{Statistical Analysis:}

Data were analyzed using R/R-Studio version 1.4.1106. Statistical tests were performed as appropriate and included the following: Student's t-test, One-Way ANOVA and Two-way ANOVA, followed by the appropriate post-hoc test, as indicated. Bars show mean \pm Standard Deviation. Differences are considered statistically significant for $p$ values less than 0.05 . All significant $p$ values are reported on graphs. 


\section{Figure Captions:}

\section{Figure 1: A High-Throughput Screen (HTS) performed in a C2C12 mouse myoblastoma Id1-Luciferase} Reporter cell lines finds Src-Family A PTKs are inherently BMPR2-Repressive, and Src-Family B PTKs are inherently BMPR2-Activating. (A) Schematic showing treatment and assessment strategy for the HTS. ;95 Cells were seeded at a density of 1,500 cells/well and subjected to siRNA knockout of one of 22,124 genes in the murine genome. After 48 hours, they were stimulated with BMP4. Two hours later, assessment of luciferase expression as well as cell viability with tryptan-blue was performed. (B) Single plot of all 22,124 genes showing the change in Id1-linked luciferin expression relative to the plate average (X-axis) and cell viability relative to the plate average (Y-axis). Red dots represent a panel of pre-selected protein tyrosine kinases (PTKs) selected from all major PTK families. (C) Change in expression of Id1-linked luciferin expression relative to background in selected receptor and non-receptor PTKs. Bars indicate standard deviation around the mean. Note that Fyn and Lck generate the greatest change in Id1 expression. (D) Change in Id1-linked luciferin expression for all Src-Family protein tyrosine kinases. Bars indicate standard deviation around the mean. Note that the overall magnitude of Id1 change correlates to the PTK's sub-family (shown in (E), reconstructed from data in [1]) with Src-A protein tyrosine kinase knockout increasing $I d 1$ and Src-B protein tyrosine kinases decreasing $I d 1$ expression.

Figure 2: Lck is expressed in the endothelium of pulmonary artery and correlates with Id1 expression. SC-RNAseq analysis in human PBMCs suggests that the expression of Bmpr2 and Id1 is low in lymphocytes which have high expression of Lck or Fyn. (A) Single molecule, RNA in situ hybridization in human lung for Lck mRNA (white), VE-Cadherin (magenta, Endothelial Marker), Acta2 (green, Smooth Muscle Marker), and DAPI (blue, nuclear marker) shows Lck mRNA co-localizes with VE-Cadherin positive cells in the lumen of pulmonary arteries. Lck-Hi, VE-Cadherin/Acta2 negative cells (arrow) likely represent lymphocytes. Bars $=20 \mu \mathrm{m}$. (B) Cultured human pulmonary artery endothelial cells (PAECs) express Lck protein in a perinuclear manner. $63 x$ objective, Bar $=50 \mu \mathrm{m}$. (C-D) Immunoblotting of protein lysate taken from human PAECs obtained from explanted human lungs from both donor controls and patients with PAH shows a correlation between Lck and Id1 expression (Id1/ $\beta$-Actin $\left.=2.6 \times \mathrm{Lck} / \beta-A c t i n+0.89, \mathrm{R}^{2}=0.68, p=0.043\right)$. (E) SC-RNAseq 
analysis of 2700 PBMCs from a healthy donor (full analysis given in Supplemental Figure 2) using the Seurat SC-RNA seq pipeline [55] shows Lck and Fyn are most highly expressed in Cd4+ and Cd8+ T-Cells, and

Natural Killer Cells. However, BMPR2 and Id1 are most highly expressed in Dendritic Cells. Note that Id2 as opposed to Id 1 or Id 3 is most highly expressed in most lymphocytes, natural killer cells, and dendritic cells. Dot size indicates the percent of cells expressing the gene of interest (cutoff of $>1$ count per cell). Average Expression is the log-scaled Z-score of counts for each gene in each cluster.

\section{Figure 3: Silencing of the Src-Family B PTK LCK in human PAECs suppresses canonical BMPR2}

signaling. (A) Human, primary pulmonary artery endothelial cells (PAECs) were transfected with si-RNA targeting LCK (si-Lck) or a nontargeting (si-NT) sequence for 6 hours. Cells were lysed at 72 hours in full media ( $2 \%$ FBS) conditions. Gene expression in the si-Lck group relative to the si-NT was determined by RT-qPCR using the relative standard method (serial 2-fold dilations of mRNA were used to make standard curves for all mRNA probes) to calculate Fold Change after normalizing to 18 s. Lck was significantly reduced (FC $=0.038 \pm$ $0.011, p<0.0001)$ as was $I d 1(F C=0.69 \pm 0.11, p=0.038)$. Snai1 was significantly increased $(F C=2.06 \pm$ $0.44, \mathrm{p}<0.0001)$. Gapdh, a common housekeeping gene, was significantly reduced by Lck knockout $(\mathrm{FC}=$ $0.58 \pm 0.11, p<0.001$ ), leading us to use 18 s as a housekeeping gene. Statistical analysis was performed by 1-Way ANOVA with Tukey's Rage Test $(F(11,36)=8000, p<0.0001)$. PAECs were transfected with either Lck or NT siRNA. At 48 hours, the cells were starved in $0.2 \%$ FBS media. 1.5 Hours prior to harvesting, the cells were treated with PBS, $20 \mathrm{ng} / \mathrm{mL}$ BMP9 or $20 \mathrm{ng} / \mathrm{mL}$ of TGF- $\beta 1$ in $0.2 \%$ FBS starvation media. After 1.5 hours of treatment and 72 hours post transfection, cells were lysed and run on SDS-PAGE. (B). Knockout of $L C K$ with si-LCK RNA suppresses total LCK protein expression at 72 hours (2-Way ANOVA, $F(2,12)=1.51, p=$ 0.26 for interaction between transfection and ligand treatment). (C). LCK knockout by siRNA significantly reduces BMP9 mediated phosopho-SMAD1 expression. The significant increase in Id1 expression induced by BMP9 treatment is significantly and almost completely attenuated after LCK knockout. There is a trend towards increased SNAIL/SLUG and phospho-SMAD3 expression in the siNT-PBS vs. siLCK-PBS conditions which was not significant. The decrease in total SMAD1 signaling seen is believed to be due to a conformational change of the SMAD1 protein in its phosphorylated state leading to unfavorable antibody binding. All $p$ values 
BMP) and transfection (siNT vs. siLCK) followed by Tukey's Range Test. In all statistical tests above, normality checks for ANOVA residuals and Levene's test for Homogeneity were carried out and assumptions met.

\section{Figure 4: Silencing of the Src-Family A PTK Fyn in human PAECs potentiates BMP9 mediated canonical}

BMPR2 signaling. (A) PAECs were transfected with either Fyn targeting (si-Fyn) or a nontargeting (si-NT) siRNA for 6 hours. 24 hours after transfection cells were starved in $0.2 \%$ FBS. At 72 hours, cells were lysed and RT-qPCR by relative standard method (the same method as Figure 3-A) was performed to determine the Fold Change (FC) in RNA expression in the si-Fyn condition relative to the si-NT condition after normalizing by 18s RNA expression. Fyn was significantly reduced (FC $=0.16 \pm 0.018, \mathrm{p}<0.0001)$ whereas $/ d 1$ was significantly increased $(F C=2.35 \pm 0.29, p<0.0001)$ as was Bmpr2 $(1.54 \pm 0.20, p<0.001)$. Statistical analysis by 1-way ANOVA. (B-C) PAECs were transfected with si-Fyn or si-NT. Cells were either maintained in full media conditions (2\% FBS) or transitioned to starvation media (0.2\% FBS) 24 hours after transfection. 1.5 hours prior to cell lysis, cells in starvation group were either treated with PBS (0.2\% FBS group) or $20 \mathrm{ng} / \mathrm{mL}$ BMP9 (0.2\% FBS+BMP9). Cells were lysed and run on SDS-PAGE. In all cases there was a significant knockdown of Fyn at 72 hours. There was no detectable change in Phospho-SMAD1 activity (pSMAD1). However, si-Fyn transfection did significantly reduce phospho-SMAD3 in the BMP9 treated cells. Finally, there was a significant increase in Id1 expression by si-Fyn transfection relative to si-NT transfection in the BMP9 treated group. All $\mathrm{p}$ values presented are derived from a 2-way ANOVA examining the interaction between treatment (2\% FBS, 0.2\% FBS + PBS, 0.2\% FBS+BMP9) and transfection (si-NT vs. si-Fyn) followed by

Tukey's Range Test. In all statistical tests above, normality checks for ANOVA residuals and Levene's test for homogeneity were carried out and assumptions met.

Figure 5: Inhibition of the Src-B PTK Lck promotes endothelial cell dysfunction, whereas inhibition of the Src-A PTK Fyn does not. (A) PAECs were transfected with either a Lck targeting (si-Lck), Fyn targeting (si-Fyn), or a nontargeting siRNA. Note that conditions were optimized for knockdown for each gene. Therefore si-Lck and s-Fyn are compared to matching control conditions (termed si-NT-L or si-NT-F) that match the transfection conditions specified for each gene. 48 hours after transfection, in full media ( $2 \%$ FBS) conditions, viable cells were transferred from 6 well plates to 24 well plates coated with Matrigel. 3 Hours after passage, 
Matrigel plugs were imaged, and tube formation was assessed with the Angiogenesis Analyzer package in

ImageJ [57]. Raw microscopic images (left) are shown alongside of analyzed images (right). Four total experiments were performed for each condition. (B) Quantification of total tube length and total tube junctions by the Angiogenesis Analyzer program finds that si-Lck is associated with a significant decrease in both total tube length and tube junctions at 3 hours. Conversely, si-Fyn promotes increased tube formation as assessed by total tube length and total tube junctions. (C) Overall, si-Lck transfection results in decreased PAEC counts over time. (D) RT-qPCR by relative standard methods in PAECs transfected with si-Lck or si-Fyn and maintained in starvation media (0.2\% FBS) for 72 hours shows a significant increased for Bax and $B c /$ mRNA relative to $18 s$ mRNA. (E) Similar results were observed for Caspase $3 / 7$ activity at 48 hours post transfection in full (2\% FBS) media. (F) Transfection with si-Lck led to increased expression of VCAM1 despite minimal reduction in Lck protein 72 hours after transfection in full (2\% FBS) media. Significance was assessed with i85 Student's T-Test.

Figure 6: Whole-transcriptome analysis shows that Lck knockout suppresses, and Fyn knockout promotes a "Pro-Endothelial" gene program (which includes the BMPR2 pathway). Conversely, Fyn knockout increases, and Lck knockout suppresses an "Inflammatory" gene program which centers on canonical NF-k $\boldsymbol{\beta}$ / RelA signaling. (A) PAECs were transfected with either Fyn (si-Fyn), Lck (si-Lck), or nontargeting siRNA (NT) for 6 hours. 24 hours post transfection, cells were starved in $0.2 \%$ FBS media. 2 Hours prior to lysis, cells were treated with either PBS or $20 \mathrm{ng} / \mathrm{mL}$ BMP9. Cells were lysed, RNA extracted, quality assessed with Bioanalyzer, sequenced, and aligned with STAR. Differentially expressed genes induced by si-Lck and si-Fyn are shown as volcano plots. (B) Normalization of counts was achieved by performing the variance stabilizing transformation of the raw count data prior to performing unsupervised hierarchical clustering and heatmap generation. One block of genes increased by si-Fyn and BMP9 but decreased by siLck correlates with canonical BMPR2 signaling (includes Bmpr2 and Id1). Several blocks upregulated by siLck and downregulated by si-Fyn correlate with inflammatory markers such as Cxc/3, II-6, Cc/8, Vcam1, and Icam1. (C) After performing differential expression analysis with DESeq2, select genes important in endothelial biology were queried. In general, si-Lck reduced markers of endothelial identity, increased markers of endothelial dysfunction, and favored NF-к $\beta$ over BMPR2 canonical signaling. (D) The set of genes co-regulated 
by si-Lck and si-Fyn were calculated. We specifically focused on genes that were increased by si-Lck and decreased by si-Fyn (Group $\mathbf{E}$, hereafter referred to as the "Inflammatory" signature) and genes that were decreased by si-Lck and increased by si-Fyn (Group F, hereafter referred to as the "Endothelial Identity" signature.) (E) Details of the 671 gene "Inflammatory" signature. Tnfaip6 and $/ 11 \mathrm{~b}$ are the most co-regulated genes in the group. Gene ontology analysis reveals this group of genes increased by si-Lck matches a response like that induced by interferon- $\beta$, interferon- $\gamma$, or lipopolysaccharide. Several computations transcription factor analysis reveal that canonical NF- $\kappa \beta$ signaling by p65 RelA plays a major role in gene regulation. (F) Details of the 481 gene "Pro-Endothelial" gene signature. Mycn, Gja5, and Lyve1 are major 710 differentially regulated genes. Gene ontology analysis centers on endothelial cell migration, adherens junction organization, and sprouting angiogenesis. No one transcription factor is revealed in computation analysis. (G) IF staining of PAECs validates that si-Lck results in increased NF- $\kappa \beta$ signaling. PAECs were transfected with si-Lck, si-Fyn, siNT-F, siNT-F, siNT-L (method as in Figure 5). 48 hours post transfection, in full media conditions, cells were fixed and stained for Lck, Fyn, phosphor-p65 RelA, and F-Actin. Cells transfected with 715 silck had a significant increase in co-localization of p-p65 RelA with the nuclear marker DAPI. Statistical analysis with 1-Way ANOVA. 


\section{Supplementary Figure Captions:}

120 Supplemental Figure 1: (A) Immunofluorescent imaging of human lung for Lck protein shows the intimal / endothelial layer exhibits mild Lck expression. Lck (red) antibody, Acta2 (smooth muscle actin, green), DAPI (blue), and autofluorescence (yellow, mainly highlights elastin and platelets). $63 x$ confocal image, bar $=50$ and $10 \mu \mathrm{m}$. (B) Densitometry of western blotting from Figure 2-C suggests a positive correlation between Id1/ $\beta$ Actin and Snail// $\beta$-Actin in healthy patients but a negative correlation in PAH patients.

Supplemental Figure 2: (A) UMAP plot of all 2700 human PBCMs after unsupervised clustering by the Seuarat single cell RNA seq pipeline. Nine distinct clusters are identified. Clusters have been annotated for cell identity based on the gene expression detailed in (2 B-C). (B) Dot plots showing expression of canonical genes known to be expressed in specific cell types which informs cluster identity. Cd3 denotes T-cells, Cd8 indicates CD8+ 130 T-Cells, KIrd1 denotes natural killer cells, Cd19 and Ms4a1 denotes B-Cells, Itgae, HLA-CQA1, and Itgax denote dendritic cells, Fcgr3a denotes monocytes, Pf4 indicates platelets, and Cd14 denotes monocytes. Dot size indicates percent expression of the gene (the number of cells in the cluster that have $>1$ counts of a given gene). Average expression is the Z-score of the log-normalized counts of a gen gene within the cluster. (C) Feature plots of all genes of interested examined in PBMCs for correlation with the clusters of Supplemental 135 Figure 2-A.

Supplemental Figure 3: Knockout of Lck in PAECs suppresses canonical BMPR2 signaling in different media conditions. PAECs were transfected with siRNA targeting Lck (si-Lck) or a nontargeting siRNA (siNT) for 6 hours. 24 hours after transfection, the cells were either starved (0.2\% FBS) or kept in full media (2\% FBS). 1.5 740 hours prior to cell lysis, the starvation cells were either treated with PBS or $20 \mathrm{ng} / \mathrm{mL}$ of BMP9. Cell lysate was run on SDS-PAGE. Phospho-SMAD1 was significantly upregulated by BMP9 treatment and was significantly reduced in the si-Lck group. Id1 was significantly reduced by siLck in both the full media condition (2\% FBS) and the BMP9 case. 2-Way ANOVA for the interaction between treatment (Full Media, Starvation Media, Starvation Media + BMP9) and transfection (si-Lck and si-NT) with Tukey's Range Test was used to determine 
745 significance. Normality checks for ANOVA residuals and Levene's test for homogeneity were carried out and assumptions met.

Supplemental Figure 4: Knockout of FYN in full media conditions increases phospho-SMAD 1 activity even in the setting of TGF- $\beta 1$ treatment in full media conditions. PAECs were transfected with siRNA targeting either

Fyn (si-Fyn) or a nontargeting siRNA (siNT) for six hours. These cells were maintained in full media (2\% FBS) for 72 hours prior to cell lysis. At 1.5 hours prior to cell lysis, cells were treated with either PBS or $10 \mathrm{ng} / \mathrm{mL}$ of TGF- $\beta 1$. There were significant reduction in Fyn for all the si-Fyn cases. Treatment with si-Fyn significantly increased phospho-SMAD1 in both the PBS and TGF- $\beta$ conditions. There was no significant change in phospho-SMAD3.

Supplemental Figure 5: Quality control metrics for the RNA seq analysis detailed in Figure 6. (A) Shows the distribution of RIN, Total Raw Reads, Clean/Raw Reads [\%], GC Content [\%], Q30 [\%], and \% of Reads Mapped by STAR. (B) Principal component analysis of all samples used in analysis shows that all conditions closely cluster together with few outliers.

Supplemental Figure 6: Detailed volcano plot of differentially expressed genes in the si-Lck cases relative to the siNT case. Note that Eln is not depicted in order to better view all genes on this plot.

Supplemental Figure 7: Detailed volcano plot of all differentially expressed genes in the si-FYN case relative 165 to the si-NT case.

Supplemental Figure 8: Detailed volcano plot of all differentially expressed genes in the BMP9 case relative to the si-NT case.

770 Supplemental Figure 9: Extension of Figure 6-E - "Inflammatory" gene signature. Extended list of genes codifferentially expressed and are increased by si-Lck and decreased si-Fyn. Because si-Lck imparts a dysfunctional endothelial phenotype, genes in this list are implicated in endothelial cell dysfunction. Genes in 
this list are increased by Lck suppression and decreased by Fyn suppression. The accompanying gene ontology analysis shows that multiple pathways are implicated that represent a response to interferon signaling,

175 lipopolysaccharide, NF- $\kappa \beta$ signaling, or neutrophil transmigration.

Supplemental Figure 10: Extension of Figure 6-F - "Pro-Endothelial" gene signature. Genes in this signature are increased by si-Fyn and decreased by si-Lck. Because si-Lck conditions generate an dysfunctional endothelial phenotype, these genes are thought to be important for endothelial health. Many (Gja5, Ace, Lyve1, Vwf, Nos3) are either canonical endothelial markers or are markers of endothelial health (as with Nos3). Fewer Gene Ontology terms are implicated, however, than with the "Inflammatory" gene signature.

Supplemental Figure 11: Genes co-differentially regulated by BMP9 and si-Lck and believed to be important for endothelial health. Because si-Lck imparts a dysfunctional phenotype to PAECs, genes that are decreased

by si-Lck are expected to be protective. Conversely, genes which are upregulated by BMP9 are believed to be important in vascular health [43]. This set contains all genes decreased in the si-Lck condition and upregulated in the BMP9 condition. Top genes include Lrrc4, Smad6, and Gja5. Gene ontology implicates these genes in multiple aspects of cardiac development, epithelial to mesenchymal transition in the endocardial cushion, and artery and lymphatic development.

Supplemental Figure 12: Genes co-differentially regulated by BMP9 and si-Lck and believed to be implicated in endothelial cell dysfunction. Because si-Lck imparts a dysfunctional phenotype to PAECs, genes in this group are upregulated by si-Lck, downregulated by BMP9, and are expected to be maladaptive in endothelial cells. Top genes include Pdk4, Cxcl2, Cxcl1, Cxcl8, Cebpd, and Tnfaip3, all of which are implicated in inflammation. 195 Top gene ontology terms involve regulation of transforming growth factor beta and beta2 production, neutrophil chemotaxis, and positive regulation of p38MAP-kinase.

Supplemental Figure 13: Genes co-regulated by BMP9 and si-Fyn and suspected to contribute to endothelial cell dysfunction. Because si-Fyn increases canonical BMPR2 signaling and either does not affect or improves metrics of endothelial cell dysfunction, genes downregulated by si-Fyn are thought to promote endothelial cell 
dysfunction. Similarly, genes decreased by BMP9 treatment are thought of as having the potential to drive a maladaptive response in endothelial cells. This shows the group of genes which are both decreased by si-Fyn and by BMP9. Top regulated genes include Cxc/8, Batf2, Mypn, Cxc/2, Cepd, and Cxc/1. By gene ontology, this set of genes is expected to contribute to B-cell homeostasis and positive regulation of leukocyte migration 305 and chemotaxis.

Supplemental Figure 14: Genes co-regulated by BMP9 and si-Fyn and expected to promote endothelial cell health. This contains the set of genes that are both upregulated by BMP9 and are also upregulated by si-Fyn. Because si-Fyn does not generate endothelial cell dysfunction, and BMP9 promotes vascular health, this set of genes is expected to promote endothelial cell health. Top upregulated genes include Gata3, Id1, Lrrc4, II21R, Gja5, Dact3, Smad6, Hey2, Gli2, and Smad9. Gene ontology analysis finds that these genes are associated with the differentiation of vascular smooth muscle, aorta morphogenesis, cardiac ventricle development, and artery morphogenesis. 


\section{References Cited:}

1. G. Manning DBW, R. Martinez, T. Hunter and S. Sudarsanam. The Protein Kinase Complement of the Human Genome. Science. 2002;298(5600):1912-6+33-34.

2. Grimminger F, Schermuly RT, Ghofrani HA. Targeting non-malignant disorders with tyrosine kinase inhibitors. Nat Rev Drug Discov. 2010;9(12):956-70. Epub 2010/12/02. doi: 10.1038/nrd3297. PubMed PMID: 21119733.

3. Rubin LJ. Primary pulmonary hypertension. N Engl J Med. 1997;336(2):111-7. Epub 1997/01/09. doi: 10.1056/NEJM199701093360207. PubMed PMID: 8988890.

4. Machado RD, Eickelberg O, Elliott CG, Geraci MW, Hanaoka M, Loyd JE, Newman JH, Phillips JA, 3rd, Soubrier F, Trembath RC, Chung WK. Genetics and genomics of pulmonary arterial hypertension. J Am Coll Cardiol. 2009;54(1 Suppl):S32-42. doi: 10.1016/j.jacc.2009.04.015. PubMed PMID: 19555857; PMCID: PMC3725550.

330 5. Rabinovitch M. Molecular pathogenesis of pulmonary arterial hypertension. J Clin Invest. 2012;122(12):4306-13. doi: 10.1172/JCI60658. PubMed PMID: 23202738; PMCID: PMC3533531.

6. Lau EMT, Giannoulatou E, Celermajer DS, Humbert M. Epidemiology and treatment of pulmonary arterial hypertension. Nat Rev Cardiol. 2017;14(10):603-14. doi: 10.1038/nrcardio.2017.84. PubMed PMID: 28593996.

335 7. Hoeper MM, Barst RJ, Bourge RC, Feldman J, Frost AE, Galie N, Gomez-Sanchez MA, Grimminger F, Grunig E, Hassoun PM, Morrell NW, Peacock AJ, Satoh T, Simonneau G, Tapson VF, Torres F, Lawrence D, Quinn DA, Ghofrani HA. Imatinib mesylate as add-on therapy for pulmonary arterial hypertension: results of the randomized IMPRES study. Circulation. 2013;127(10):1128-38. doi: 10.1161/CIRCULATIONAHA.112.000765. PubMed PMID: 23403476.

340 8. Pankey EA, Thammasiboon S, Lasker GF, Baber S, Lasky JA, Kadowitz PJ. Imatinib attenuates monocrotaline pulmonary hypertension and has potent vasodilator activity in pulmonary and systemic vascular beds in the rat. Am J Physiol Heart Circ Physiol. 2013;305(9):H1288-96. doi: 10.1152/ajpheart.00329.2013. PubMed PMID: 23997103; PMCID: PMC3840242.

9. Rasheed W, Flaim B, Seymour JF. Reversible severe pulmonary hypertension secondary to dasatinib in

a patient with chronic myeloid leukemia. Leuk Res. 2009;33(6):861-4. doi: 10.1016/j.leukres.2008.09.026. PubMed PMID: 18986702.

10. Montani D, Bergot E, Gunther S, Savale L, Bergeron A, Bourdin A, Bouvaist H, Canuet M, Pison C, Macro M, Poubeau P, Girerd B, Natali D, Guignabert C, Perros F, O'Callaghan DS, Jais X, Tubert-Bitter P, Zalcman G, Sitbon O, Simonneau G, Humbert M. Pulmonary arterial hypertension in patients treated by dasatinib. Circulation. 2012;125(17):2128-37. doi: 10.1161/CIRCULATIONAHA.111.079921. PubMed PMID: 22451584.

11. Guignabert C, Phan C, Seferian A, Huertas A, Tu L, Thuillet R, Sattler C, Le Hiress M, Tamura Y, Jutant EM, Chaumais MC, Bouchet S, Maneglier B, Molimard M, Rousselot P, Sitbon O, Simonneau G, Montani D, Humbert M. Dasatinib induces lung vascular toxicity and predisposes to pulmonary hypertension. J Clin Invest. 2016;126(9):3207-18. doi: 10.1172/JCI86249. PubMed PMID: 27482885; PMCID: PMC5004960. 
355 12. Hickey PM, Thompson AA, Charalampopoulos A, Elliot CA, Hamilton N, Kiely DG, Lawrie A, Sabroe I, Condliffe R. Bosutinib therapy resulting in severe deterioration of pre-existing pulmonary arterial hypertension. Eur Respir J. 2016;48(5):1514-6. doi: 10.1183/13993003.01004-2016. PubMed PMID: 27660511.

13. Quilot FM, Georges M, Favrolt N, Beltramo G, Foignot C, Grandvuillemin A, Montani D, Bonniaud P, Camus P. Pulmonary hypertension associated with ponatinib therapy. Eur Respir J. 2016;47(2):676-9. doi: 10.1183/13993003.01110-2015. PubMed PMID: 26743481.

14. Swords R, Mahalingam D, Padmanabhan S, Carew J, Giles F. Nilotinib: optimal therapy for patients with chronic myeloid leukemia and resistance or intolerance to imatinib. Drug Des Devel Ther. 2009;3:89-101. Epub 2009/11/19. doi: 10.2147/dddt.s3069. PubMed PMID: 19920925; PMCID: PMC2769239.

15. Gover-Proaktor A, Granot G, Pasmanik-Chor M, Pasvolsky O, Shapira S, Raz O, Raanani P, Leader A. Bosutinib, dasatinib, imatinib, nilotinib, and ponatinib differentially affect the vascular molecular pathways and functionality of human endothelial cells. Leuk Lymphoma. 2019;60(1):189-99. Epub 2018/05/10. doi: 10.1080/10428194.2018.1466294. PubMed PMID: 29741440.

16. Dasgupta SK, Le A, Vijayan KV, Thiagarajan P. Dasatinib inhibits actin fiber reorganization and promotes endothelial cell permeability through RhoA-ROCK pathway. Cancer Med. 2017;6(4):809-18. Epub 2017/03/21. doi: 10.1002/cam4.1019. PubMed PMID: 28316141; PMCID: PMC5387130.

17. Phan C, Jutant EM, Tu L, Thuillet R, Seferian A, Montani D, Huertas A, Bezu JV, Breijer F, Vonk Noordegraaf A, Humbert M, Aman J, Guignabert C. Dasatinib increases endothelial permeability leading to pleural effusion. Eur Respir J. 2018;51(1). Epub 2018/01/20. doi: 10.1183/13993003.01096-2017. PubMed PMID: 29348177.

375 18. Cornet L, Khouri C, Roustit M, Guignabert C, Chaumais MC, Humbert M, Revol B, Despas F, Montani D, Cracowski JL. Pulmonary arterial hypertension associated with protein kinase inhibitors: a pharmacovigilancepharmacodynamic study. Eur Respir J. 2019;53(5). doi: 10.1183/13993003.02472-2018. PubMed PMID: 30846469.

19. Ochoa D, Jarnuczak AF, Vieitez C, Gehre M, Soucheray M, Mateus A, Kleefeldt AA, Hill A, Garcia-Alonso L, Stein F, Krogan NJ, Savitski MM, Swaney DL, Vizcaino JA, Noh KM, Beltrao P. The functional landscape of the human phosphoproteome. Nat Biotechnol. 2020;38(3):365-73. Epub 2019/12/11. doi: 10.1038/s41587-0190344-3. PubMed PMID: 31819260; PMCID: PMC7100915.

20. Shah NH, Lobel M, Weiss A, Kuriyan J. Fine-tuning of substrate preferences of the Src-family kinase Lck revealed through a high-throughput specificity screen. Elife. 2018;7. doi: 10.7554/eLife.35190. PubMed PMID: 29547119; PMCID: PMC5889215.

21. Zhu N, Gonzaga-Jauregui $C$, Welch CL, Ma L, Qi H, King AK, Krishnan U, Rosenzweig EB, Ivy DD, Austin ED, Hamid R, Nichols WC, Pauciulo MW, Lutz KA, Sawle A, Reid JG, Overton JD, Baras A, Dewey F, Shen Y, Chung WK. Exome Sequencing in Children With Pulmonary Arterial Hypertension Demonstrates Differences Compared With Adults. Circ Genom Precis Med. 2018;11(4):e001887. doi: 10.1161/CIRCGEN.117.001887. PubMed PMID: 29631995; PMCID: PMC5896781.

22. Andruska A, Spiekerkoetter E. Consequences of BMPR2 Deficiency in the Pulmonary Vasculature and Beyond: Contributions to Pulmonary Arterial Hypertension. Int J Mol Sci. 2018;19(9). doi: 10.3390/ijms19092499. PubMed PMID: 30149506. 
23. Frump AL, Lowery JW, Hamid R, Austin ED, de Caestecker M. Abnormal trafficking of endogenously expressed BMPR2 mutant allelic products in patients with heritable pulmonary arterial hypertension. PLoS One. 2013;8(11):e80319. doi: 10.1371/journal.pone.0080319. PubMed PMID: 24224048; PMCID: PMC3818254.

24. Prewitt AR, Ghose S, Frump AL, Datta A, Austin ED, Kenworthy AK, de Caestecker MP. Heterozygous null bone morphogenetic protein receptor type 2 mutations promote SRC kinase-dependent caveolar trafficking defects and endothelial dysfunction in pulmonary arterial hypertension. J Biol Chem. 2015;290(2):960-71. doi: 10.1074/jbc.M114.591057. PubMed PMID: 25411245; PMCID: PMC4294523.

25. Dannewitz Prosseda S, Tian X, Kuramoto K, Boehm M, Sudheendra D, Miyagawa K, Zhang F, SolowCordero D, Saldivar JC, Austin ED, Loyd JE, Wheeler L, Andruska A, Donato M, Wang L, Huebner K, Metzger RJ, Khatri P, Spiekerkoetter E. Fragile Histidine Triad (FHIT), a Novel Modifier Gene in Pulmonary Arterial Hypertension. Am J Respir Crit Care Med. 2018. doi: 10.1164/rccm.201712-25530C. PubMed PMID: 30107138.

26. Zilberberg $L$, ten Dijke $P$, Sakai LY, Rifkin DB. A rapid and sensitive bioassay to measure bone morphogenetic protein activity. BMC Cell Biol. 2007;8:41. Epub 2007/09/21. doi: 10.1186/1471-2121-8-41. PubMed PMID: 17880711; PMCID: PMC2094707.

27. Pullamsetti SS, Berghausen EM, Dabral S, Tretyn A, Butrous E, Savai R, Butrous G, Dahal BK, Brandes RP, Ghofrani HA, Weissmann N, Grimminger F, Seeger W, Rosenkranz S, Schermuly RT. Role of Src tyrosine kinases in experimental pulmonary hypertension. Arterioscler Thromb Vasc Biol. 2012;32(6):1354-65. doi: 10.1161/ATVBAHA.112.248500. PubMed PMID: 22516066.

28. Kim DJ, Norden PR, Salvador J, Barry DM, Bowers SLK, Cleaver O, Davis GE. Src- and Fyn-dependent apical membrane trafficking events control endothelial lumen formation during vascular tube morphogenesis. ) 15 PLoS One. 2017;12(9):e0184461. doi: 10.1371/journal.pone.0184461. PubMed PMID: 28910325; PMCID: PMC5598984.

29. Baumgart B, Guha M, Hennan J, Li J, Woicke J, Simic D, Graziano M, Wallis N, Sanderson T, Bunch RT. In vitro and in vivo evaluation of dasatinib and imatinib on physiological parameters of pulmonary arterial hypertension. Cancer Chemother Pharmacol. 2017;79(4):711-23. doi: 10.1007/s00280-017-3264-2. PubMed PMID: 28283735.

30. Chakraborty AK, Weiss A. Insights into the initiation of TCR signaling. Nat Immunol. 2014;15(9):798807. doi: 10.1038/ni.2940. PubMed PMID: 25137454; PMCID: PMC5226627.

31. Richard KC, Bertolesi GE, Dunfield LD, McMaster CR, Nachtigal MW. TSAd interacts with Smad2 and Smad3. Biochem Biophys Res Commun. 2006;347(1):266-72. doi: 10.1016/j.bbrc.2006.06.068. PubMed PMID: 16806069.

32. David L, Mallet C, Mazerbourg S, Feige JJ, Bailly S. Identification of BMP9 and BMP10 as functional activators of the orphan activin receptor-like kinase 1 (ALK1) in endothelial cells. Blood. 2007;109(5):1953-61. doi: 10.1182/blood-2006-07-034124. PubMed PMID: 17068149.

33. Gimbrone MA, Jr., Garcia-Cardena G. Endothelial Cell Dysfunction and the Pathobiology of 330 Atherosclerosis. Circ Res. 2016;118(4):620-36. Epub 2016/02/20. doi: 10.1161/CIRCRESAHA.115.306301. PubMed PMID: 26892962; PMCID: PMC4762052.

34. Travaglini KJ, Nabhan AN, Penland L, Sinha R, Gillich A, Sit RV, Chang S, Conley SD, Mori Y, Seita J, Berry GJ, Shrager JB, Metzger RJ, Kuo CS, Neff N, Weissman IL, Quake SR, Krasnow MA. A molecular cell atlas of the 
human lung from single-cell RNA sequencing. Nature. 2020;587(7835):619-25. Epub 2020/11/20. doi: 10.1038/s41586-020-2922-4. PubMed PMID: 33208946; PMCID: PMC7704697.

35. Evrard SM, Lecce L, Michelis KC, Nomura-Kitabayashi A, Pandey G, Purushothaman KR, d'Escamard V, Li JR, Hadri L, Fujitani K, Moreno PR, Benard L, Rimmele P, Cohain A, Mecham B, Randolph GJ, Nabel EG, Hajjar $\mathrm{R}$, Fuster V, Boehm M, Kovacic JC. Endothelial to mesenchymal transition is common in atherosclerotic lesions and is associated with plaque instability. Nat Commun. 2016;7:11853. Epub 2016/06/25. doi: 10.1038/ncomms11853. PubMed PMID: 27340017; PMCID: PMC4931033.

36. Kuleshov MV, Jones MR, Rouillard AD, Fernandez NF, Duan Q, Wang Z, Koplev S, Jenkins SL, Jagodnik KM, Lachmann A, McDermott MG, Monteiro CD, Gundersen GW, Ma'ayan A. Enrichr: a comprehensive gene set enrichment analysis web server 2016 update. Nucleic Acids Res. 2016;44(W1):W90-7. Epub 2016/05/05. doi: 10.1093/nar/gkw377. PubMed PMID: 27141961; PMCID: PMC4987924.

37. Kwon AT, Arenillas DJ, Worsley Hunt R, Wasserman WW. oPOSSUM-3: advanced analysis of regulatory motif over-representation across genes or ChIP-Seq datasets. G3 (Bethesda). 2012;2(9):987-1002. Epub 2012/09/14. doi: 10.1534/g3.112.003202. PubMed PMID: 22973536; PMCID: PMC3429929.

38. Han H, Cho JW, Lee S, Yun A, Kim H, Bae D, Yang S, Kim CY, Lee M, Kim E, Lee S, Kang B, Jeong D, Kim Y, Jeon HN, Jung $\mathrm{H}$, Nam S, Chung M, Kim JH, Lee I. TRRUST V2: an expanded reference database of human and mouse transcriptional regulatory interactions. Nucleic Acids Res. 2018;46(D1):D380-D6. Epub 2017/11/01. doi: 10.1093/nar/gkx1013. PubMed PMID: 29087512; PMCID: PMC5753191.

39. Wang Z, Civelek M, Miller CL, Sheffield NC, Guertin MJ, Zang C. BART: a transcription factor prediction tool with query gene sets or epigenomic profiles. Bioinformatics. 2018;34(16):2867-9. Epub 2018/04/03. doi: 10.1093/bioinformatics/bty194. PubMed PMID: 29608647; PMCID: PMC6084568.

55 40. Looney AP, Han R, Stawski L, Marden G, Iwamoto M, Trojanowska M. Synergistic Role of Endothelial ERG and FLI1 in Mediating Pulmonary Vascular Homeostasis. Am J Respir Cell Mol Biol. 2017;57(1):121-31. Epub 2017/03/02. doi: 10.1165/rcmb.2016-02000C. PubMed PMID: 28248553; PMCID: PMC5516275.

41. Shah AV, Birdsey GM, Peghaire C, Pitulescu ME, Dufton NP, Yang Y, Weinberg I, Osuna Almagro L, Payne L, Mason JC, Gerhardt H, Adams RH, Randi AM. The endothelial transcription factor ERG mediates Angiopoietin-1-dependent control of Notch signalling and vascular stability. Nat Commun. 2017;8:16002. Epub 2017/07/12. doi: 10.1038/ncomms16002. PubMed PMID: 28695891; PMCID: PMC5508205.

42. Plumitallo S, Ruiz-Llorente L, Langa C, Morini J, Babini G, Cappelletti D, Scelsi L, Greco A, Danesino C, Bernabeu C, Olivieri C. Functional analysis of a novel ENG variant in a patient with hereditary hemorrhagic telangiectasia (HHT) identifies a new Sp1 binding-site. Gene. 2018;647:85-92. Epub 2018/01/07. doi: 10.1016/j.gene.2018.01.007. PubMed PMID: 29305977.

43. Long L, Ormiston ML, Yang X, Southwood M, Graf S, Machado RD, Mueller M, Kinzel B, Yung LM, Wilkinson JM, Moore SD, Drake KM, Aldred MA, Yu PB, Upton PD, Morrell NW. Selective enhancement of endothelial BMPR-II with BMP9 reverses pulmonary arterial hypertension. Nat Med. 2015;21(7):777-85. doi: 10.1038/nm.3877. PubMed PMID: 26076038; PMCID: PMC4496295.

370 44. Neet K, Hunter T. Vertebrate non-receptor protein-tyrosine kinase families. Genes Cells. 1996;1(2):14769. Epub 1996/02/01. doi: 10.1046/j.1365-2443.1996.d01-234.x. PubMed PMID: 9140060. 
45. Courtney AH, Lo WL, Weiss A. TCR Signaling: Mechanisms of Initiation and Propagation. Trends Biochem Sci. 2018;43(2):108-23. doi: 10.1016/j.tibs.2017.11.008. PubMed PMID: 29269020; PMCID: PMC5801066.

375 46. Herazo-Maya JD, Noth I, Duncan SR, Kim S, Ma SF, Tseng GC, Feingold E, Juan-Guardela BM, Richards TJ, Lussier Y, Huang Y, Vij R, Lindell KO, Xue J, Gibson KF, Shapiro SD, Garcia JG, Kaminski N. Peripheral blood mononuclear cell gene expression profiles predict poor outcome in idiopathic pulmonary fibrosis. Sci Transl Med. 2013;5(205):205ra136. doi: 10.1126/scitranslmed.3005964. PubMed PMID: 24089408; PMCID: PMC4175518.

800 47. Matache C, Onu A, Stefanescu M, Tanaseanu S, Dragomir C, Dolganiuc A, Szegli G. Dysregulation of p56lck inase in patients with systemic lupus erythematosus. Autoimmunity. 2001;34(1):27-38.

48. Hurst LA, Dunmore BJ, Long L, Crosby A, Al-Lamki R, Deighton J, Southwood M, Yang X, Nikolic MZ, Herrera B, Inman GJ, Bradley JR, Rana AA, Upton PD, Morrell NW. TNFalpha drives pulmonary arterial hypertension by suppressing the BMP type-II receptor and altering NOTCH signalling. Nat Commun. 2017;8:14079. doi: 10.1038/ncomms14079. PubMed PMID: 28084316; PMCID: PMC5241886.

49. Ness JK, Snyder KM, Tapinos N. Lck tyrosine kinase mediates beta1-integrin signalling to regulate Schwann cell migration and myelination. Nat Commun. 2013;4:1912. doi: 10.1038/ncomms2928. PubMed PMID: 23715271; PMCID: PMC3674276.

50. Spiekerkoetter E, Tian X, Cai J, Hopper RK, Sudheendra D, Li CG, El-Bizri N, Sawada H, Haghighat R, Chan R, Haghighat L, de Jesus Perez V, Wang L, Reddy S, Zhao M, Bernstein D, Solow-Cordero DE, Beachy PA, Wandless TJ, Ten Dijke P, Rabinovitch M. FK506 activates BMPR2, rescues endothelial dysfunction, and reverses pulmonary hypertension. J Clin Invest. 2013;123(8):3600-13. doi: 10.1172/JCI65592. PubMed PMID: 23867624; PMCID: PMC3726153.

51. Chen PI, Cao A, Miyagawa K, Tojais NF, Hennigs JK, Li CG, Sweeney NM, Inglis AS, Wang L, Li D, Ye M, Feldman BJ, Rabinovitch M. Amphetamines promote mitochondrial dysfunction and DNA damage in pulmonary hypertension. JCl Insight. 2017;2(2):e90427. Epub 2017/02/01. doi: 10.1172/jci.insight.90427. PubMed PMID: 28138562; PMCID: PMC5256132.

52. Dobin A, Davis CA, Schlesinger F, Drenkow J, Zaleski C, Jha S, Batut P, Chaisson M, Gingeras TR. STAR: ultrafast universal RNA-seq aligner. Bioinformatics. 2013;29(1):15-21. Epub 2012/10/30. doi: 10.1093/bioinformatics/bts635. PubMed PMID: 23104886; PMCID: PMC3530905.

53. Love MI, Huber W, Anders S. Moderated estimation of fold change and dispersion for RNA-seq data with DESeq2. Genome Biol. 2014;15(12):550. Epub 2014/12/18. doi: 10.1186/s13059-014-0550-8. PubMed PMID: 25516281; PMCID: PMC4302049.

54. Chen EY, Tan CM, Kou Y, Duan Q, Wang Z, Meirelles GV, Clark NR, Ma'ayan A. Enrichr: interactive and collaborative HTML5 gene list enrichment analysis tool. BMC Bioinformatics. 2013;14:128. Epub 2013/04/17. doi: 10.1186/1471-2105-14-128. PubMed PMID: 23586463; PMCID: PMC3637064.

55. Stuart T, Butler A, Hoffman P, Hafemeister C, Papalexi E, Mauck WM, 3rd, Hao Y, Stoeckius M, Smibert P, Satija R. Comprehensive Integration of Single-Cell Data. Cell. 2019;177(7):1888-902 e21. Epub 2019/06/11. doi: 10.1016/j.cell.2019.05.031. PubMed PMID: 31178118; PMCID: PMC6687398. 
)10 56. Wang F, Flanagan J, Su N, Wang LC, Bui S, Nielson A, Wu X, Vo HT, Ma XJ, Luo Y. RNAscope: a novel in situ RNA analysis platform for formalin-fixed, paraffin-embedded tissues. J Mol Diagn. 2012;14(1):22-9. Epub 2011/12/15. doi: 10.1016/j.jmoldx.2011.08.002. PubMed PMID: 22166544; PMCID: PMC3338343.

57. Carpentier G, Berndt S, Ferratge S, Rasband W, Cuendet M, Uzan G, Albanese P. Angiogenesis Analyzer for ImageJ - A comparative morphometric analysis of "Endothelial Tube Formation Assay" and "Fibrin Bead )15 Assay". Sci Rep. 2020;10(1):11568. Epub 2020/07/16. doi: 10.1038/s41598-020-67289-8. PubMed PMID: 32665552 ; PMCID: PMC7360583. 
A.

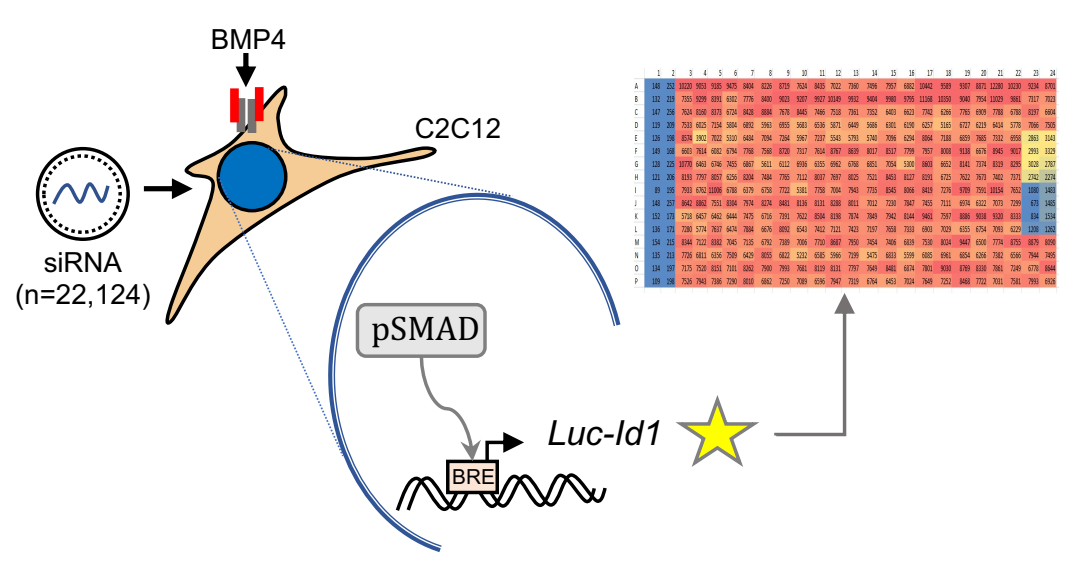

B.

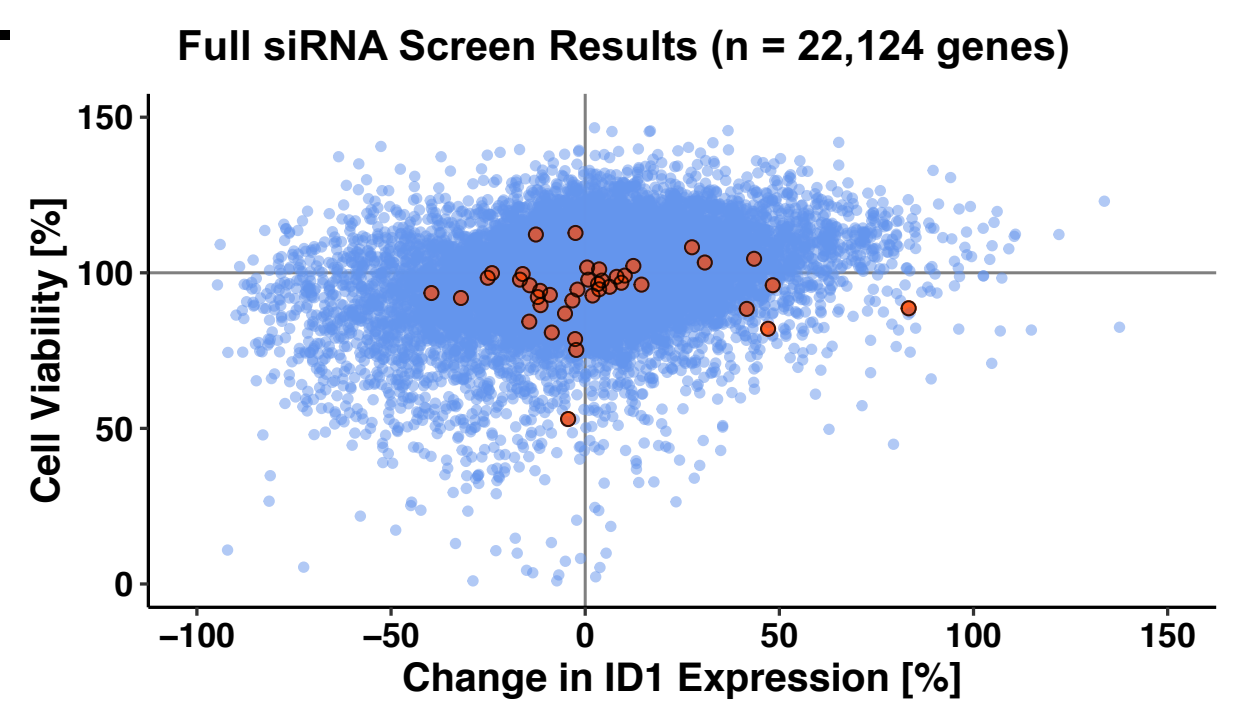

C.

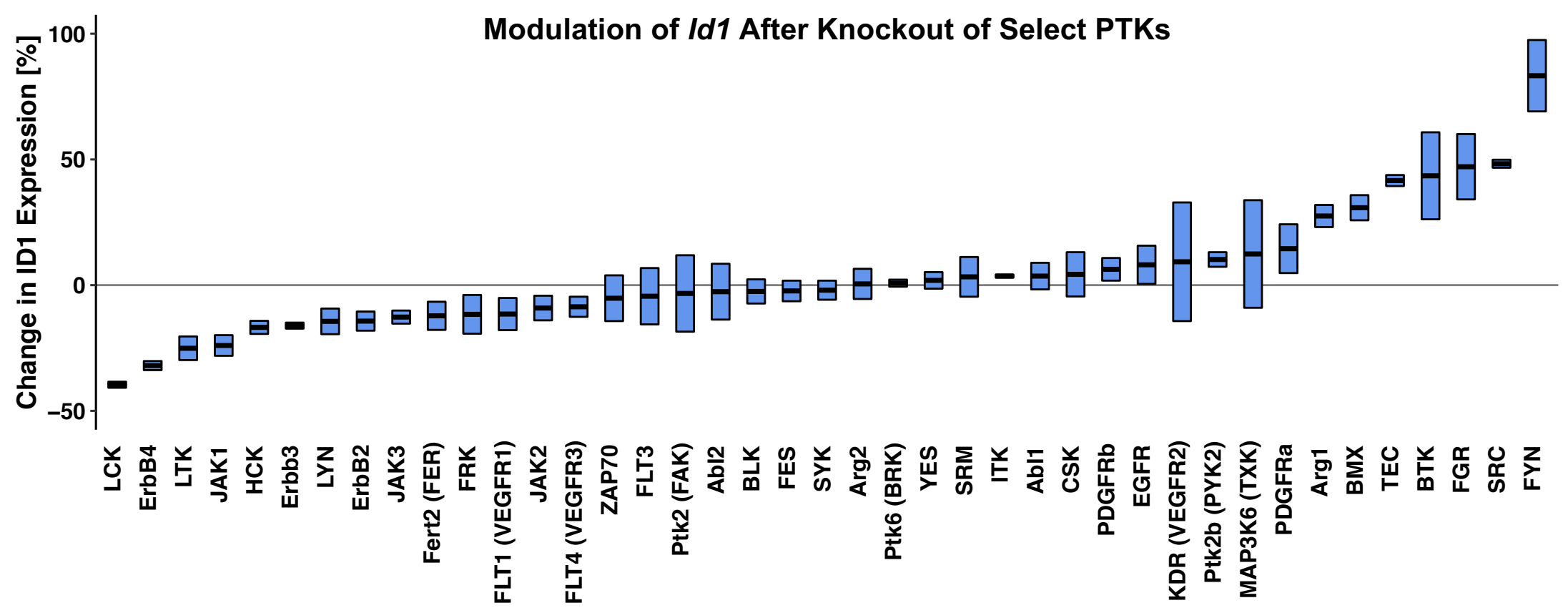

D.

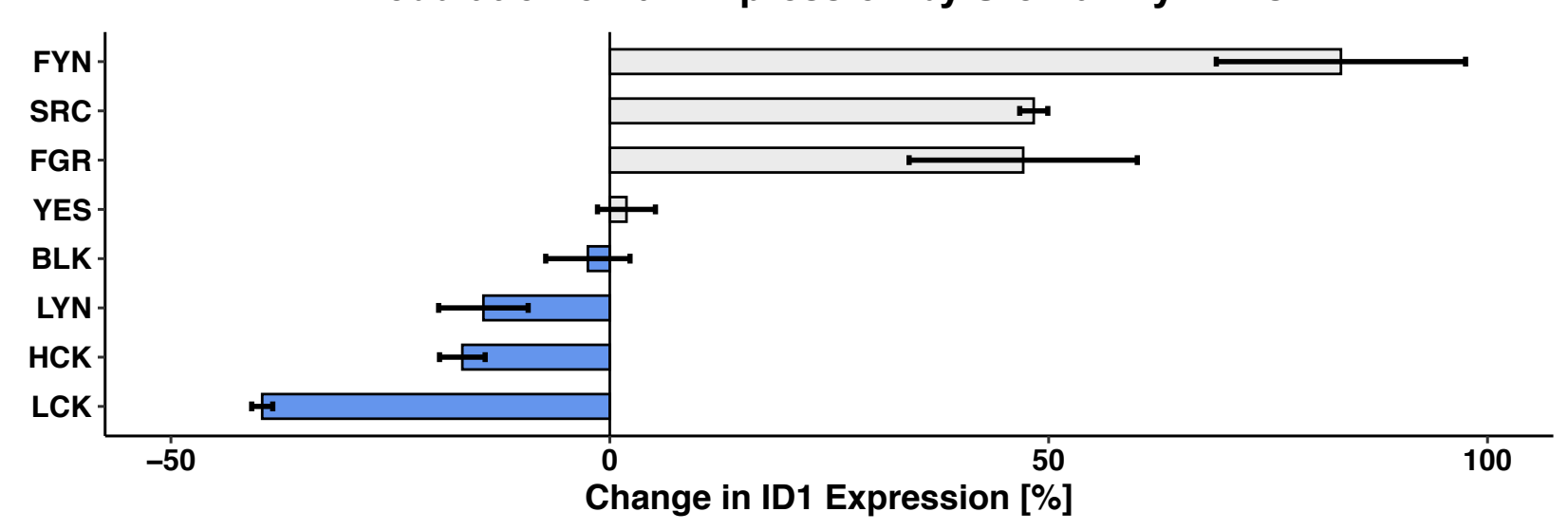

E.

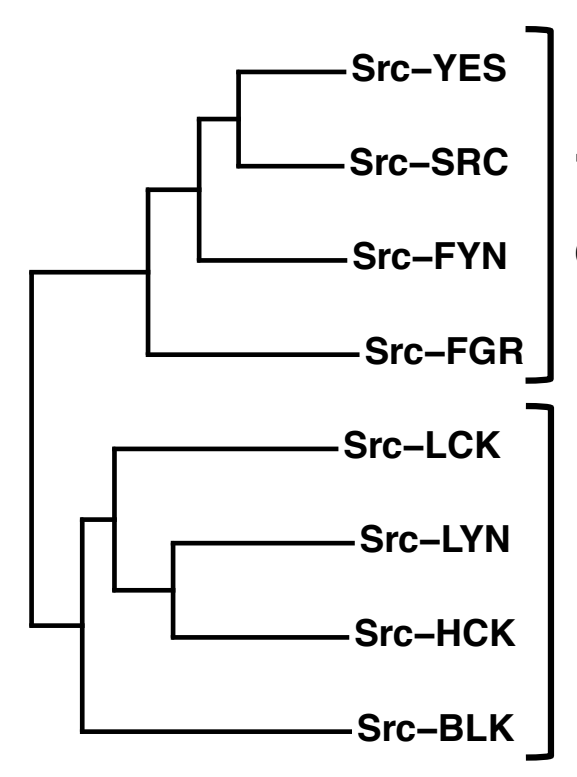


A.

SiFYN vs. SiNT

$F(3,16)=1730, p=2.65 \mathrm{e}-20$

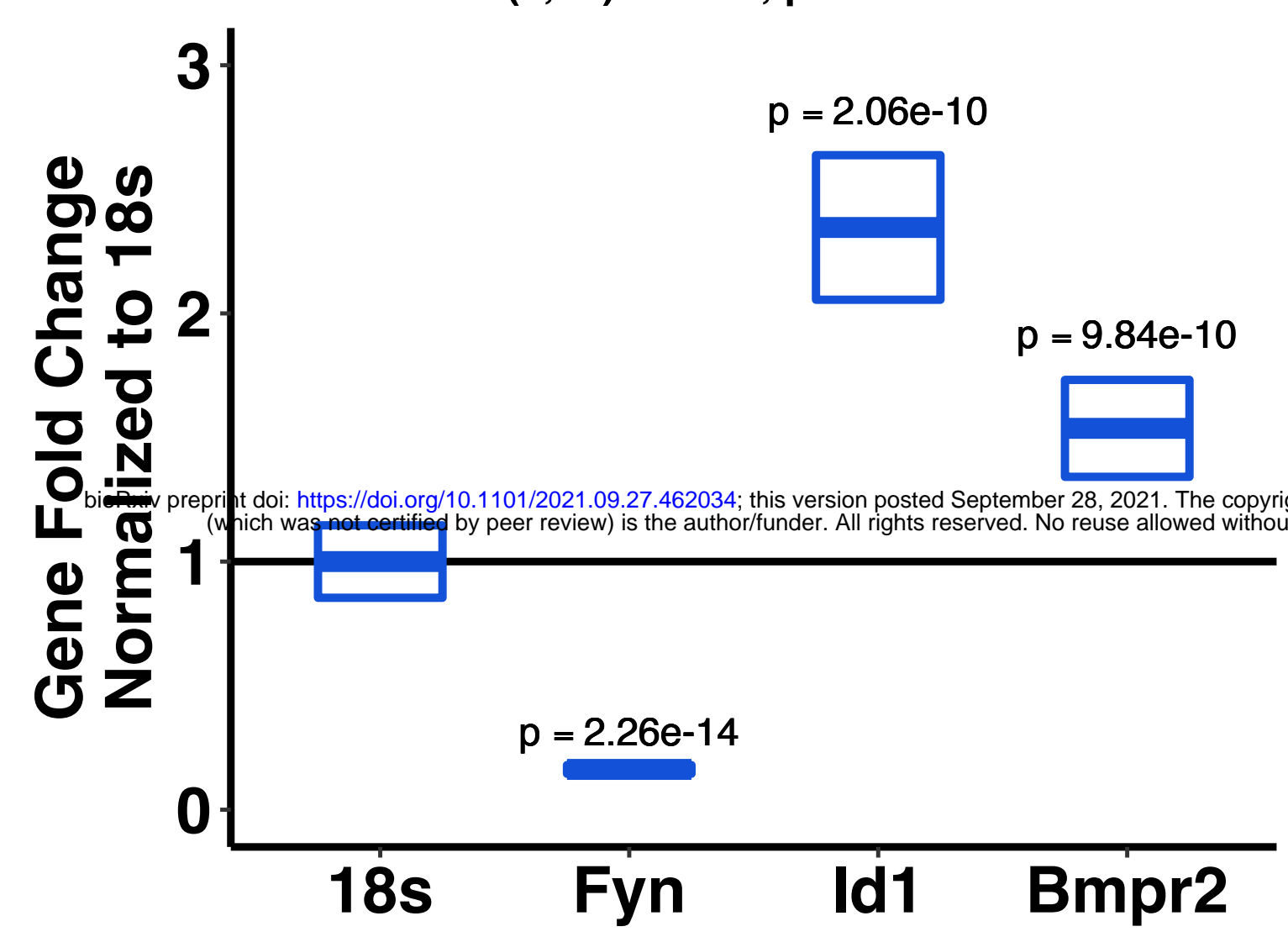

C.

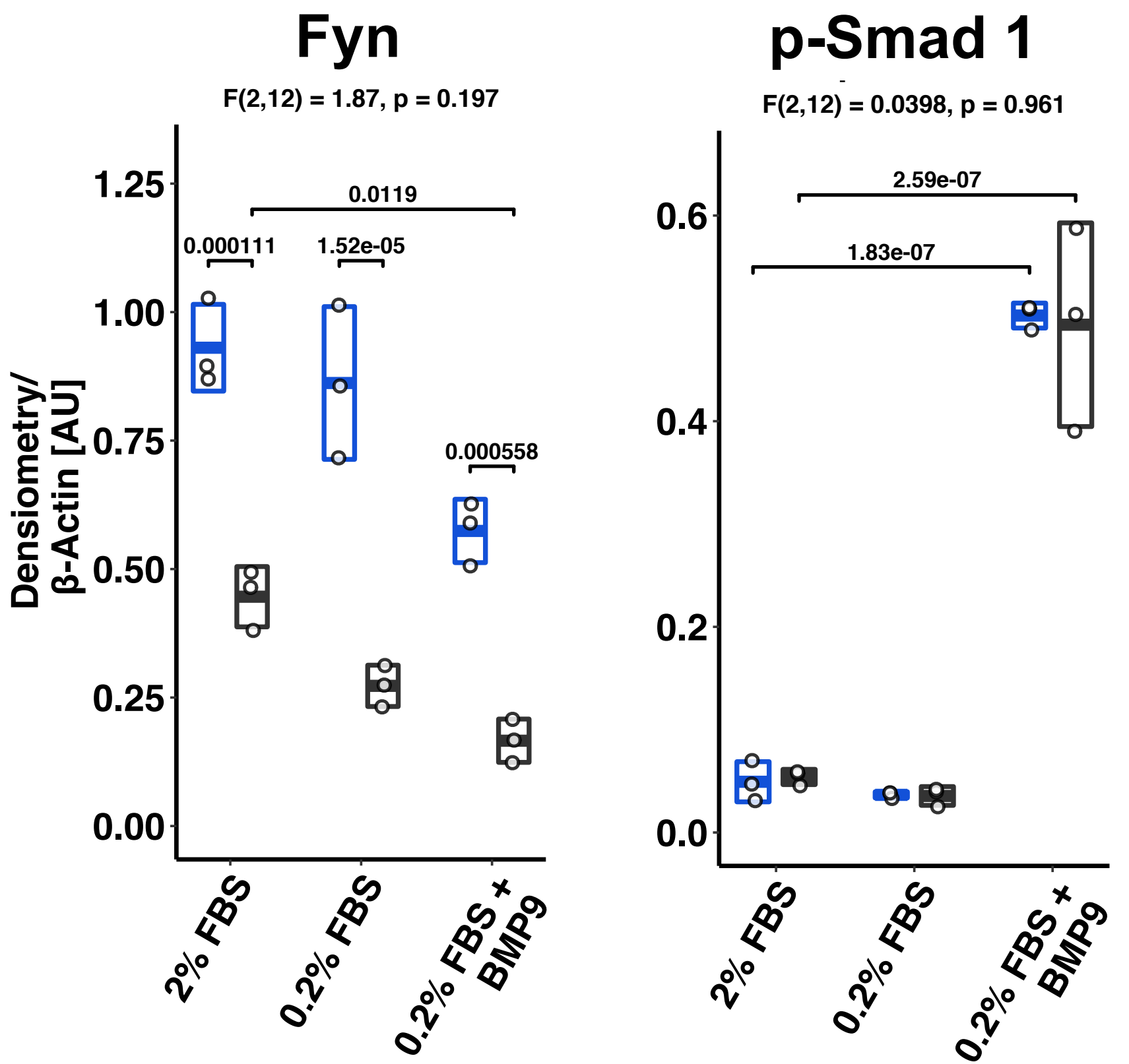

Fyn PSMAD1.

$\beta$-Actin
B.

$2 \%$ FBS

$0.2 \%$ FBS+BMP9

\begin{tabular}{|c|c|c|c|c|c|}
\hline siNT & siFyn & siNT & siFyn & siNT & siFyn \\
\hline
\end{tabular}

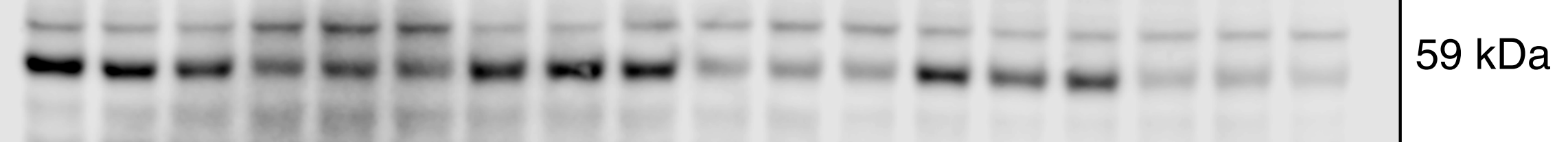

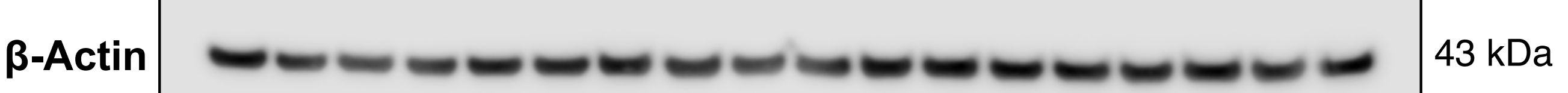

브배배배 $+\begin{aligned} & 60 \mathrm{kDa} \\ & 48 \mathrm{kDa}\end{aligned}$
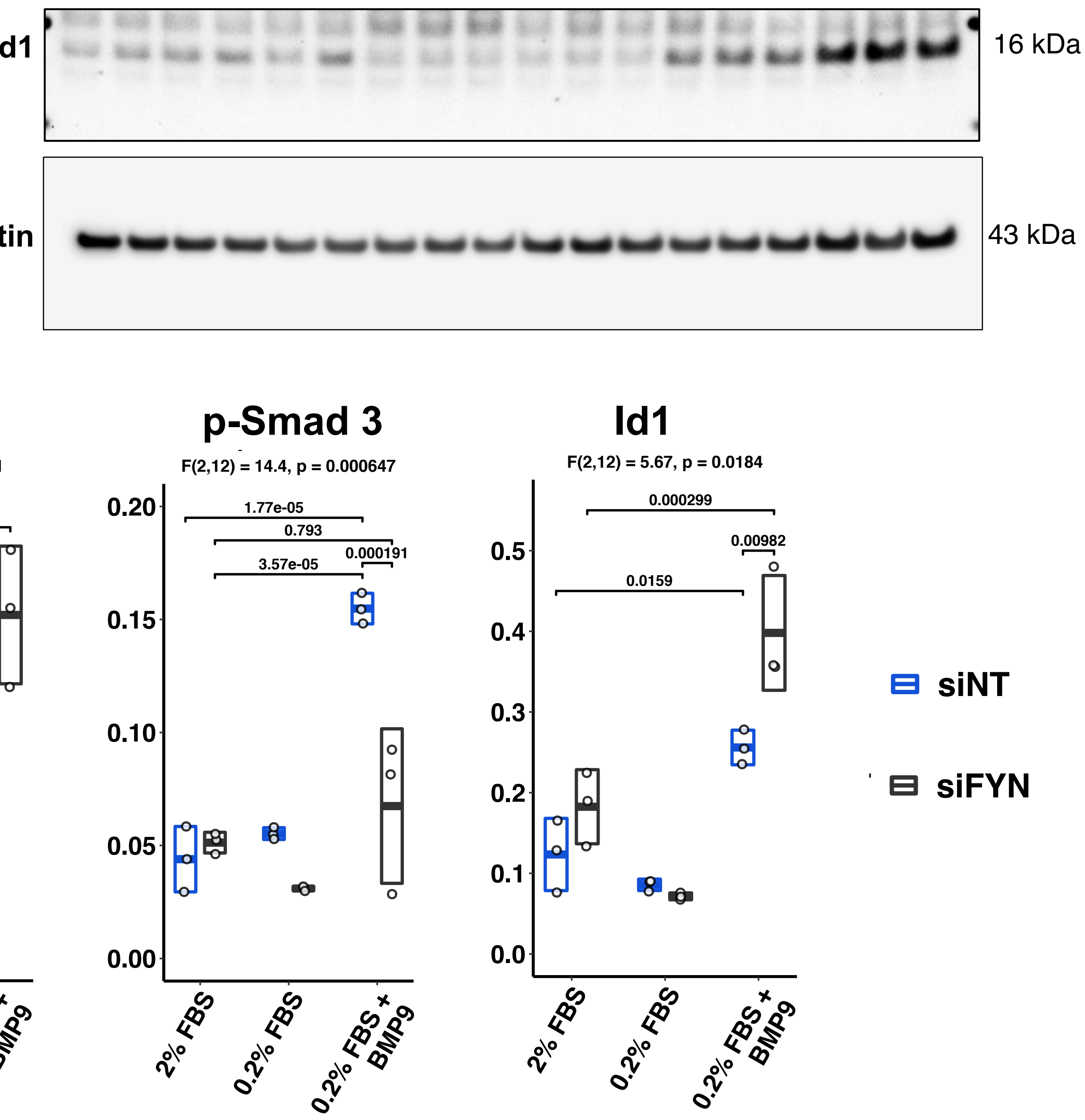

Id1

$F(2,12)=5.67, p=0.0184$
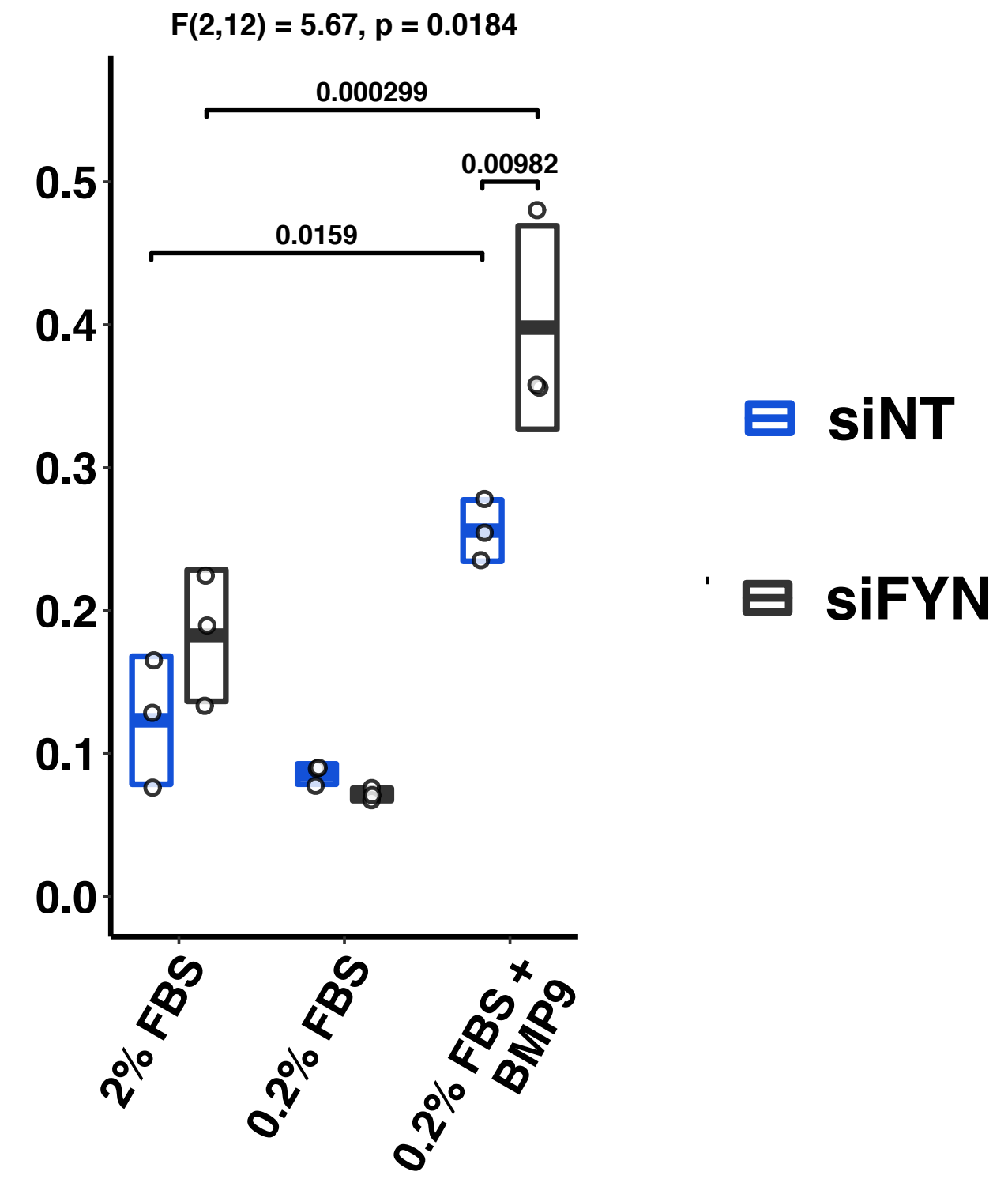


\section{A.}
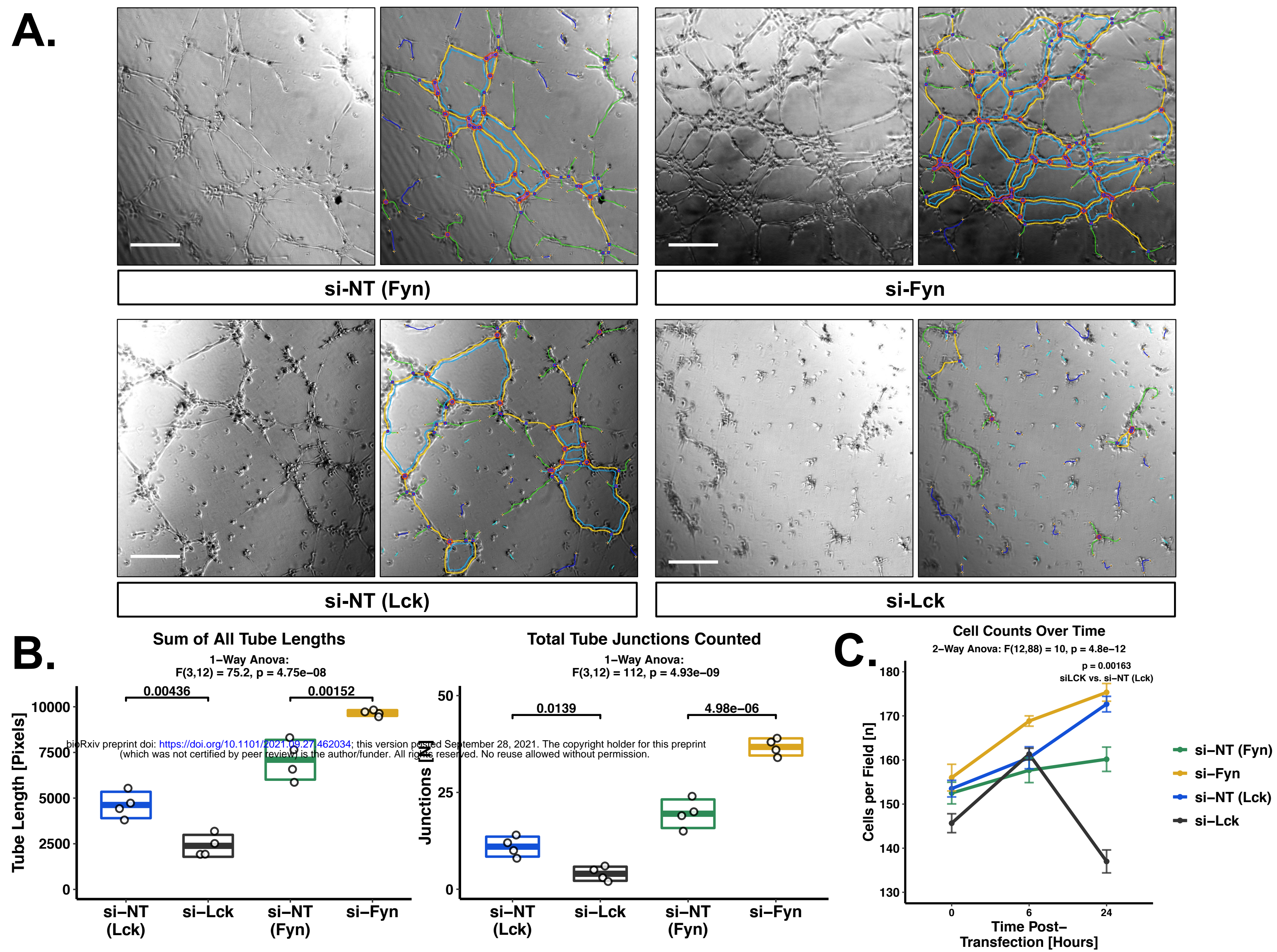

D

Bax/Bcl Gene Expression

$F(4,18)=40.8, p=8.54 \mathrm{e}-09$
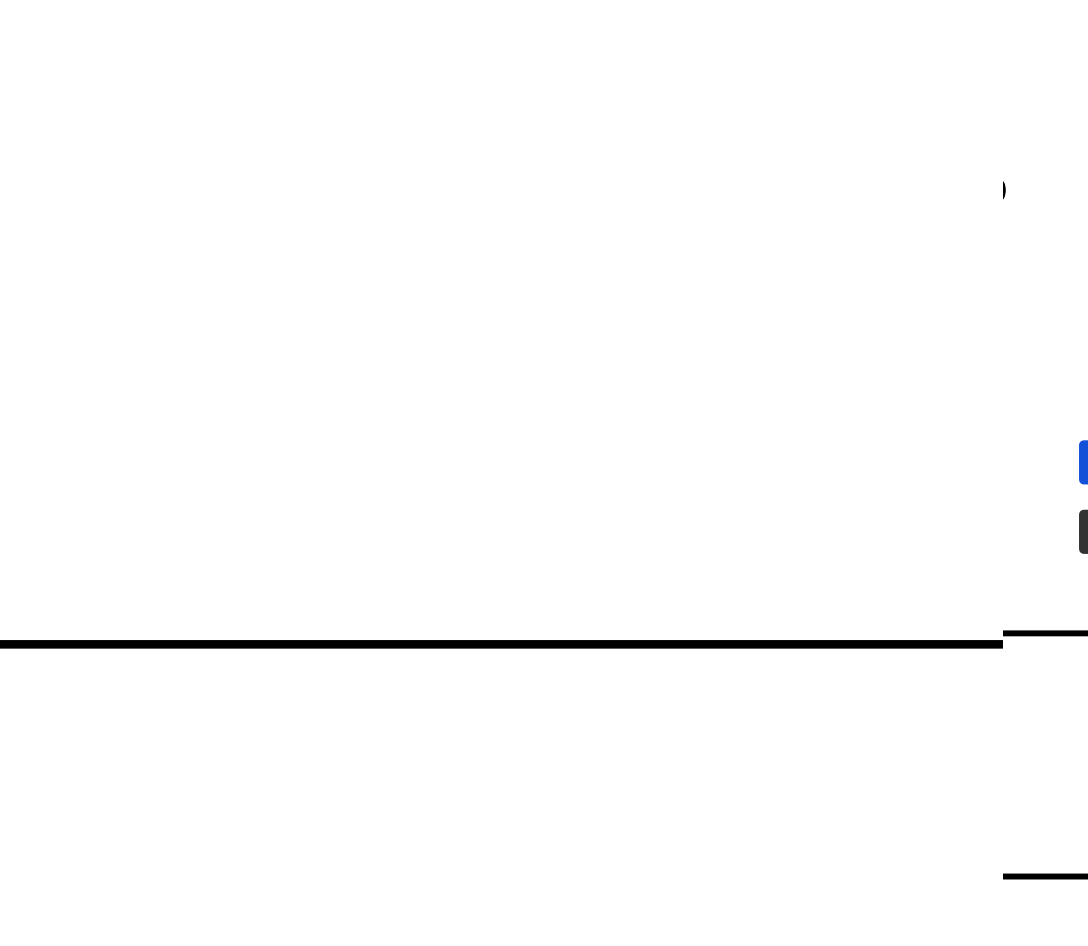

F.

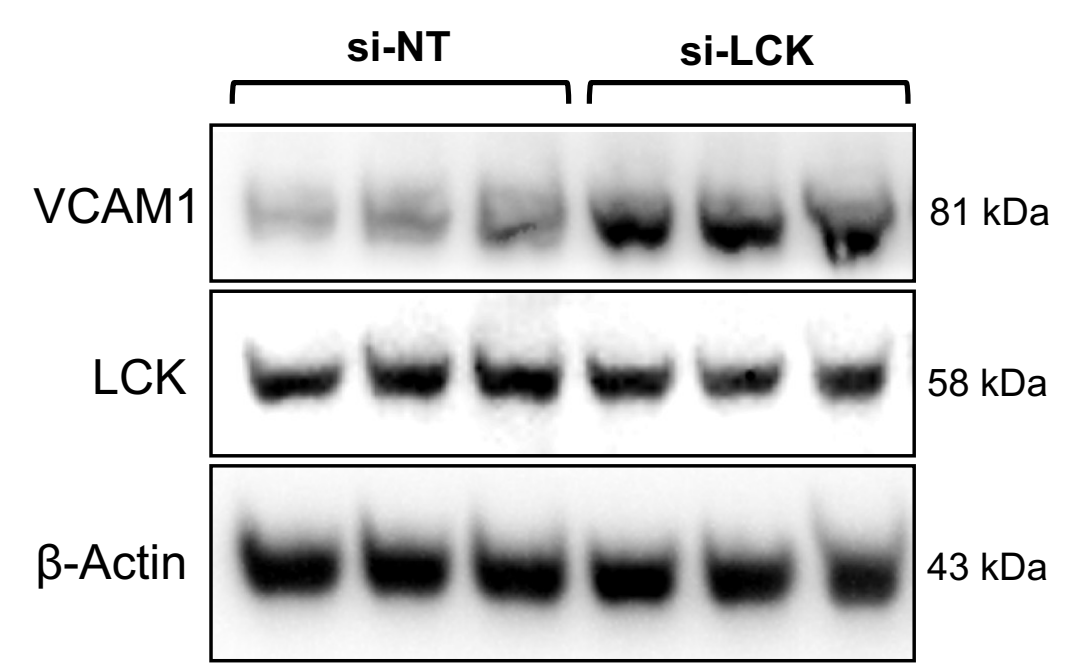

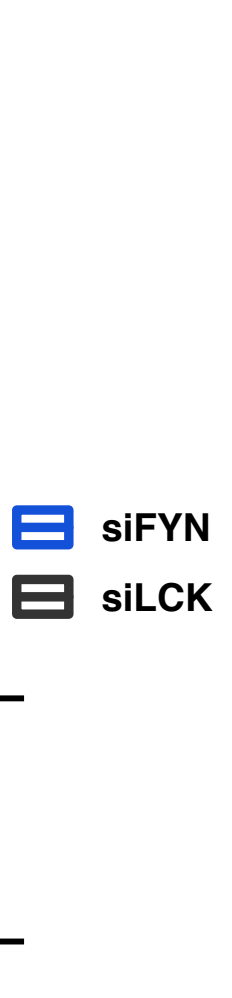

E.

si-Fyn

$F(2,42)=1.06, p=0.356$

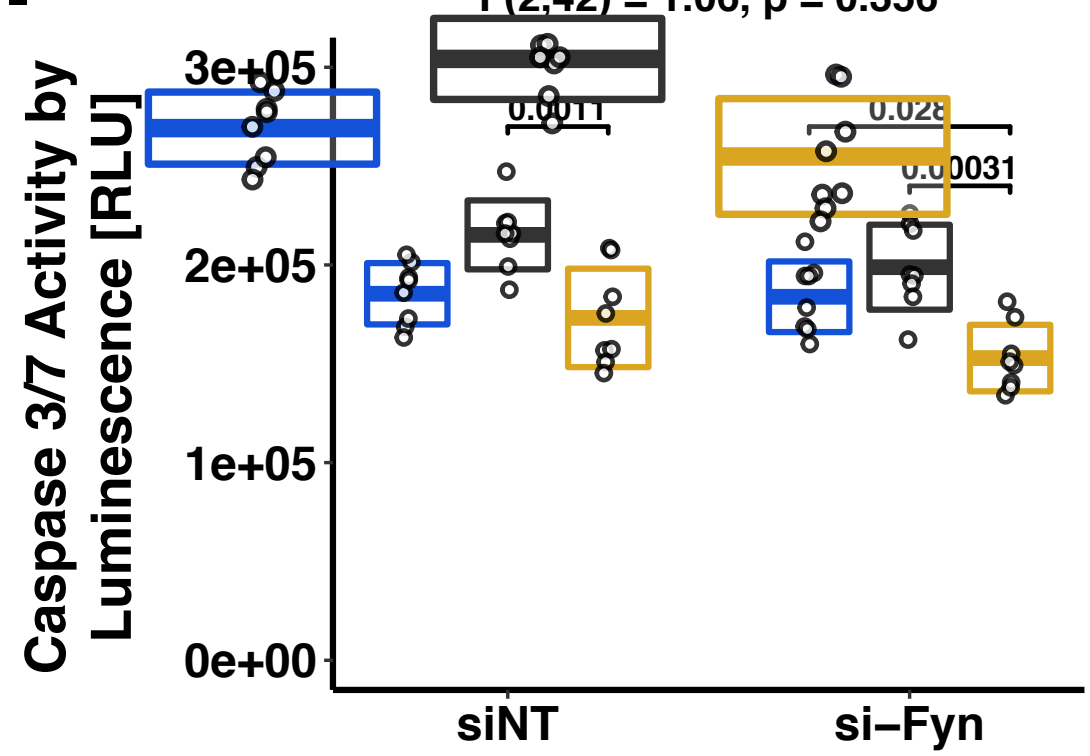

si-Lck

$F(2,23)=2.83, p=0.0798$

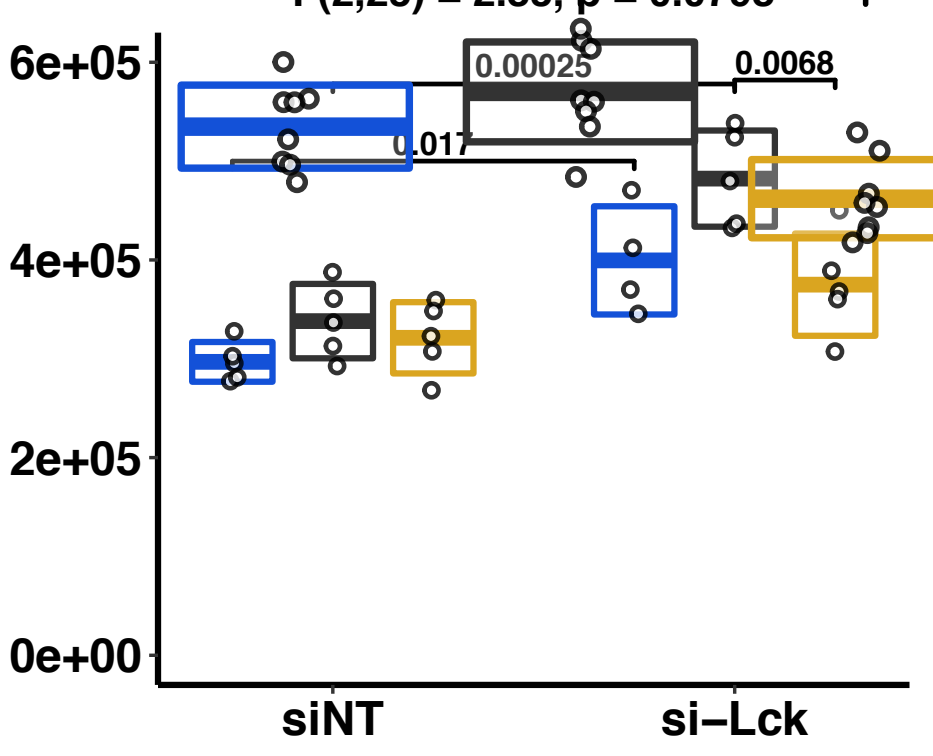

曰 2\% FBS 日 $0.2 \%$ FBS $\boxminus 0.2 \%$ FBS + BMP9

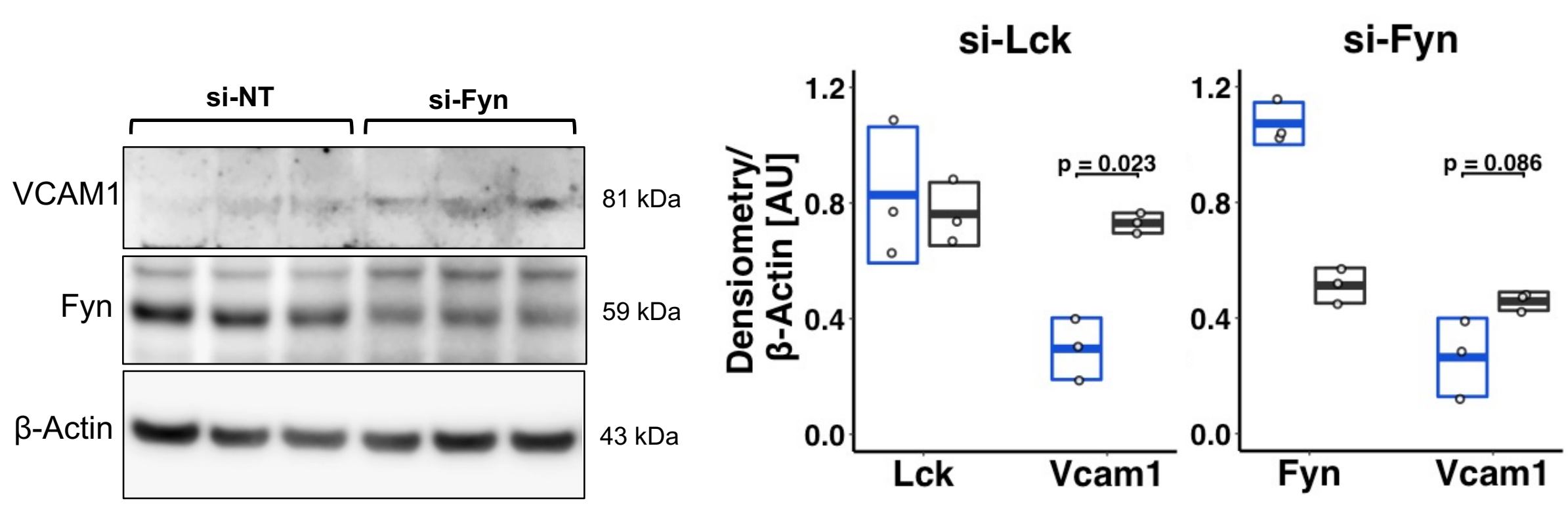


